\title{
$\mathrm{LiCl}$ 促进的多官能团格氏试剂的制备及应用研究进展
}

\author{
刘雨燕 ${ }^{a}$ 方烨汶 $*, a$ 张 莉 ${ }^{a, b}$ 金小平 $*, c$ 李瑞丰 $*, b$ 朱帅汝 ${ }^{a}$ \\ 高浩其 ${ }^{a}$ 房江华 ${ }^{a}$ 夏勤波 ${ }^{a}$ \\ ( ${ }^{a}$ 宁波工程学院化学工程学院 宁波 315016) \\ $\left({ }^{b}\right.$ 太原理工大学化学化工学院 太原 030024) \\ (c 浙江医药高等专科学校基础部 宁波 315100)
}

\begin{abstract}
摘要 自格氏试剂发现以来, 含多官能团的格氏试剂合成一直是有机镁合成化学研究的热点和难点. 重点综述了 Knochel 小组以氯化锂为添加剂, 成功地通过三类常用的格氏试剂制备方法(金属镁和有机卤化物的直接氧化加成、卤 素-镁交换、 $\mathrm{C}-\mathrm{H}$ 键的镁化)合成了一系列含多官能团的格氏试剂. 氯化锂的引入不但解决了官能团的兼容性问题, 还 提高了格氏试剂的反应活性，从而极大拓展了格氏试剂在合成化学中的应用. 对氯化锂促进型格氏试剂合成的局限和 研究前景也进行了探讨和展望.
\end{abstract}

关键词＼cjkstart格氏试剂；多官能团；氯化锂；卤素-镁交换; 镁化反应

\section{Advances in LiCl-Promoted Preparation of Polyfunctional Grignard Reagents and the Applications}

\author{
Liu, Yuyan ${ }^{a} \quad$ Fang, Yewen*,a ${ }^{*}$ Zhang, Li ${ }^{a, b} \quad$ Jin, Xiaoping ${ }^{*, c} \quad$ Li, Ruifeng ${ }^{*, b}$ \\ Zhu, Shuairu $^{a} \quad$ Gao, Haoq $^{a} \quad$ Fang, Jianghua $^{a} \quad$ Xia, Qinbo $^{a}$ \\ $\left({ }^{a}\right.$ School of Chemical Engineering, Ningbo University of Technology, Ningbo 315016) \\ $\left({ }^{b}\right.$ College of Chemistry and Chemical Engineering, Taiyuan University of Technology, Taiyuan 030024) \\ ( ${ }^{c}$ Department of Basic Education, Zhejiang Pharmaceutical College, Ningbo 315100)
}

\begin{abstract}
Since the discovery of Grignard reagents, the synthesis of highly functionalized organomagnesium compounds has been hot but difficult project. In the presence of $\mathrm{LiCl}$, a series of polyfunctional Grignard reagents were successfully prepared by Knochel group via direct oxidative addition of magnesium to organic halides, halogen-magnesium exchange reaction, or direct magnesiation of $\mathrm{C}-\mathrm{H}$ bond. In the presence of $\mathrm{LiCl}$, the issue of functional group compatibilities was addressed and the reactivity was also enhanced. Consequently, the applications of Grignard reagents in synthetic chemistry were greatly expanded. The limitations and potential research areas for $\mathrm{LiCl}$-promoted synthesis of Grignard reagents are also discussed.
\end{abstract}

Keywords Grignard reagent; polyfunctional group; lithium chloride; halogen-Mg exchange; magnesiation reaction

$\mathrm{RMgX}(\mathrm{X}$ 为卤素, $\mathrm{R}$ 为烃基)和 $\mathrm{RMgR}$ 等有机镁试 剂，通常称之为格氏试剂(Grignard Reagent), 由法国化 学家格林尼亚(Francois Agust Victor Grignard)于 1900 年 发现 ${ }^{[1]}$. 由于格氏试剂具有合成原料经济易得、合成方 法直接简便、参与反应活性高等特点，格氏试剂成为有 机合成中常用有机金属试剂 ${ }^{[2]}$. 同时, 发现于 20 世纪 70
年代的 Kumada-Corriu 反应掀起了格氏试剂在过渡金属 催化的 $\mathrm{C}-\mathrm{C}$ 偶联反应中应用的热潮 ${ }^{[3]}$.

由于金属美的电负性介于金属锂和金属锌之间 (Scheme 1), 因此, 有机镁试剂的反应活性也处于相应 的有机锂试剂和有机锌试剂之间 ${ }^{[4]}$. 和有机锂试剂相比, 有机镁试剂有更高的稳定性，在参与反应时有更好的化

\footnotetext{
*E-mail: nbut.fang@gmail.com

Received March 3, 2014; revised April 2, 2014; published online April 18, 2014

Project supported by the National Natural Science Foundation of China (No. 21202090), the Ningbo Science and Technology Innovation Team (No. 2011B82002), the Zhejiang Provincial Natural Science Foundation of China (Nos. LY12B02001, LQ13B010004), the Ningbo Natural Science Foundation (Nos. 2011A610123, 2012A610123), the Qianjiang Talents Project (B) (No. 2013R10076), and the Xinmiao Talents Program (No. 2011R422008).

国家自然科学基金(No. 21202090)、宁波市科技创新团队(No. 2011B82002)、浙江省自然科学基金(Nos. LY12B02001, LQ13B010004)、宁波市自然科 学基金(Nos. 2011A610123, 2012A610123)、钱江人才计(B) (No. 2013R10076)、新苗人才计划(No. 2011R422008)资助项目.
} 
学和立体选择性. 相比于有机锌试剂, 有机镁试剂的合 成则要容易得多; 同时由于更高的反应活性, 使得格氏 试剂参与的反应条件更温和、效率更高效. 正是基于上 述这些特点, 化学家们围绕格氏试剂的制备、应用和机 理开展了大量卓有成效的研究, 使有机镁化学在有机合 成中占据重要研究地位.

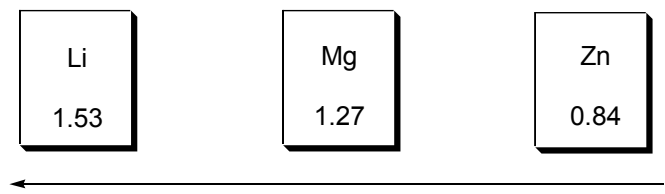

反应活性

Scheme 1

自 20 世纪初对格氏试剂的合成研究开展以来, 已 发展出多类格氏试剂的合成方法 ${ }^{[5]}$. 但常用的且具有较 好底物普适性的合成方法有三种: (1)金属美和有机卤化 物的直接氧化加成(Direct Oxidative Addition of Magnesium to Organic Halides); (2)卤素-镁交换反应(HalogenMagnesium Exchange Reaction); (3) $\mathrm{C}-\mathrm{H}$ 键的直接镁化 反应(Direct Magnesiation of C-H Bond). 但传统的方法 在制备含多官能团的格氏试剂时往往会遇到各种局限, 从而限制了格氏试剂在有机合成中的应用. 因此, 如何 合成含多官能团的有机镁试剂成为有机镁化学研究领 域中一个重要课题.

格氏试剂的反应性能在很大程度上依赖于反应温 度: 只有活泼的亲电试剂, 如醛和大多数酮可以和格氏 试剂在 $0{ }^{\circ} \mathrm{C}$ 以下发生反应. 如果能在较低温度实现格氏 试剂的合成, 那么解决格氏试剂和各类官能团的兼容问 题就成为了可能. 德国慕尼黑工业大学的 Knochel 等 ${ }^{[6]}$ 小组以 $\mathrm{LiCl}$ 作为促进剂, 开创性地实现了含多官能团 格氏试剂及其它有机金属试剂的合成(Scheme 2). 根据 各类反应条件和底物官能团兼容性的要求, 通过选用合 适的格氏试剂制备方法, 合成了一系列含多官能团的格 氏试剂，极大拓展了格氏反应的应用范围. 由于 Knochel 小组在格氏试剂合成与应用方面的杰出贡献, 通常将含 $\mathrm{LiCl}$ 的格氏试剂称之为 Konchel 型格氏试剂 (Knochel-Type Grignard Reagent)或 TurboGrignard Reagent. 本文将重点综述 $\mathrm{LiCl}$ 促进的格氏试剂的合成与 应用研究进展, 并展望有机镁化学研究的新领域.

\section{$1 \mathrm{LiCl}$ 促进的格氏试剂合成}

\section{$1.1 \mathrm{LiCl}$ 在卤素-镁交换反应中的应用}

早在 1931 年, Prévost ${ }^{[7]}$ 就运用溴-镁交换反应，通过 乙基溴化镁和肉桂基澳反应, 以 $14 \%$ 的产率合成了肉桂 基溴化镁(Eq. 1). 之后虽有类似的零星报道 ${ }^{[8]}$, 但一直
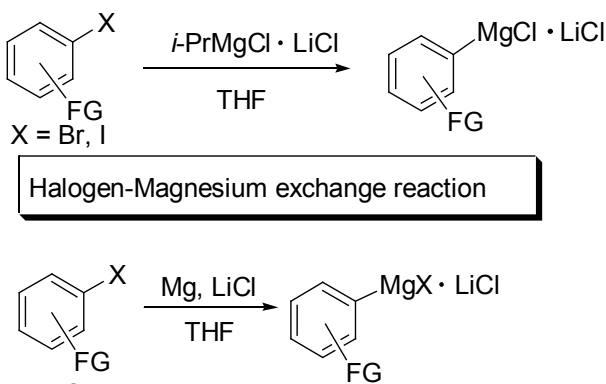

$\mathrm{X}=\mathrm{Cl}, \mathrm{Br}, \mathrm{I}$

Direct oxidative addition of magnesium to organic halides

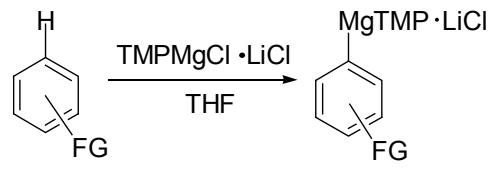

Direct magnesiation of $\mathrm{C}-\mathrm{H}$ bond

Scheme 2

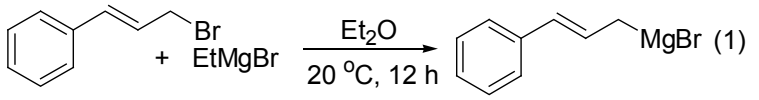

都没有得到系统研究.

1998 年, Knochel 小组 ${ }^{[9]}$ 首次报道了低温反应条件 下, 异丙基氯化镁 $(i-\mathrm{PrMgCl})$ 、异丙基澳化镁 $(i-\mathrm{PrMgBr})$ 、 二异丙基镁 $\left(i-\mathrm{Pr}_{2} \mathrm{Mg}\right)$ 和苯基氯化镁 $(\mathrm{PhMgCl})$ 是适用于 卤素-镁交换反应的几种有机镁试剂. 运用溴-镁、碘-镁 交换反应，一系列含多官能团的芳香类、芳香杂环类、 烯基、烷基等格氏试剂得以高效合成(Scheme 3), 丰富 了格氏试剂的种类和运用 ${ }^{[4,5]}$.
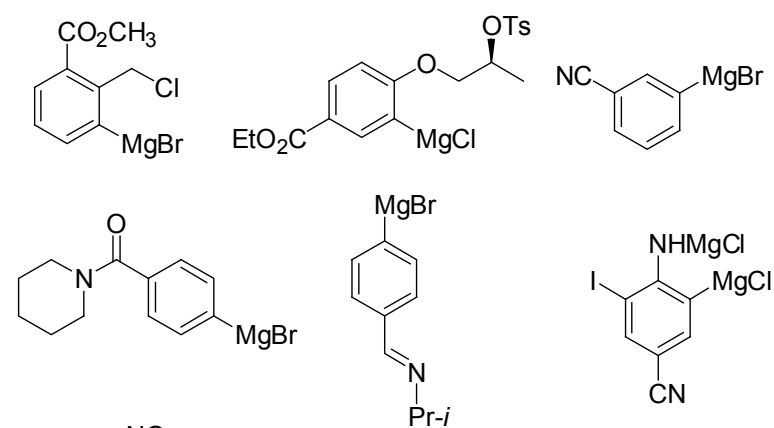<smiles>N#Cc1cc(Cl)c(NOCl)c(I)c1</smiles><smiles>CCOC(=O)c1ccc([N+](=O)[O-])c([N+](=O)[O-])c1</smiles>

$\mathrm{MgBr}$<smiles>COc1c(F)c(F)nc(F)c1F</smiles><smiles>CCOC(=CC(=[Nb]Br)c1ccc(C#N)cc1)OCC</smiles><smiles>CC1(C)OC(=O)C([N+](=O)[O-])=C(c2ccccc2)O1</smiles>

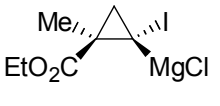

Scheme 3 
2004 年, Knochel 小组 ${ }^{[10]}$ 在 $\mathrm{Li}$ (acac) 可以加快 $\mathrm{I} / \mathrm{Zn}$ 交换反应的研究基础上, 系统地研究了各类锂盐对 $\mathrm{Br} / \mathrm{Mg}$ 交换反应速率的影响. 研究结果表明: $\mathrm{LiCl}$ 可以 最为有效地加快 $\mathrm{Br} / \mathrm{Mg}$ 交换反应速度 ${ }^{[11]}$. 如 Scheme 4 所示, 运用高活性的异丙基氯化镁 $(i-\mathrm{PrMgCl})$ 和富电子 的对甲氧基溴苯进行 $\mathrm{Br} / \mathrm{Mg}$ 交换反应时, 反应速度很 慢. 当加入当量的 $\mathrm{LiCl}$ 作为添加剂时, 在相同的反应温 度和时间下，反应转化率可以从 $18 \%$ 上升到 $84 \%$.

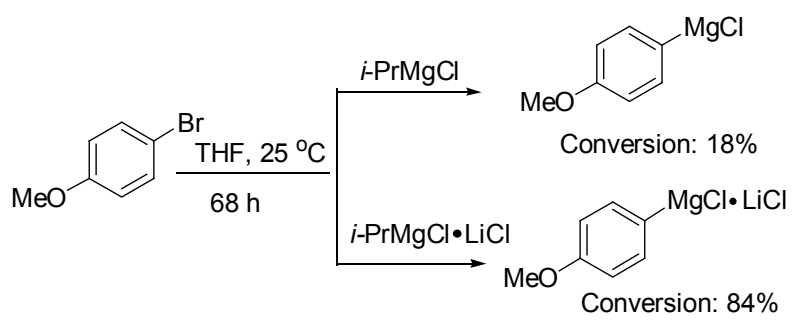

Scheme 4

尽管 $i-\mathrm{PrMgCl} \cdot \mathrm{LiCl}$ 在 $\mathrm{Br} / \mathrm{Mg}$ 交换反应中表现出了 很好的反应活性, 但对于富电子的芳基溴代物仍存在反 应效率低的问题. 在后续研究中, Knochel 小组 ${ }^{[12]}$ 引 了反应活性更高的二烷基美氯化锂作为卤素-镁交换反 应试剂. 通过对照实验可以看出：在相同反应温度下， 二异丙基镁氯化锂比异丙基氯化镁氯化锂的反应活性 要高得多(Eq. 2). 对于选用的三个富电子溴代芳烃, 选 用异丙基氯化镁氯化锂作为溴-镁交换反应试剂时, 转 化率都在 40\%以下; 而二异丙基镁氯化锂可以使对应的 溴-镁交换反应转化率达到 $96 \%$ 以上. 由此可以看出, 反应活性更高的二烷基镁氯化锂进一步拓展了卤素-镁 交换反应在富电子格氏试剂合成中的应用.

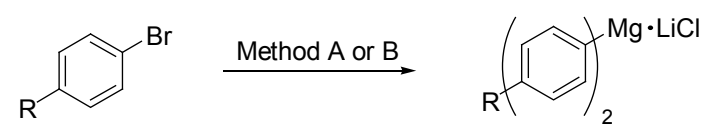

Method A: $i$ - $\mathrm{PrMgCl} \cdot \mathrm{LiCl}\left(1.05\right.$ equiv.), $25^{\circ} \mathrm{C}$, THF;

Method B: $i-\mathrm{Pr}_{2} \mathrm{Mg} \cdot \mathrm{LiCl}\left(0.525\right.$ equiv.), $25^{\circ} \mathrm{C}$, THF/Dioxane $(V: V=9: 1)$<smiles>COc1ccc(N(C)C(Cl)(Cl)Cl)cc1</smiles>

$24 \mathrm{~h}$ $24 \mathrm{~h}$,

Method A: 31\%;

Method A: $36 \%$

Method B: > 99\%

$48 \mathrm{~h}$,

Method B: > 99\%

Method A: $16 \%$;

Method B: > 96\%

二烷基镁氯化锂除了在富电子卤代烃的卤素-镁交 换反应中体现出高反应活性以外, 还体现在烯基卤代物 的卤素-镁交换反应上. 以 $i-\mathrm{PrMgCl} \cdot \mathrm{LiCl}$ 作为卤素-镁 反应的交换试剂时, 需要用高反应活性的烯基碘代 物 ${ }^{[13]}$; 而用二仲丁基镁氯化锂作为交换试剂时, 烯基溴 代物即可高效发生交换反应(Scheme 5$)^{[12]}$. 值得一提的
是，卤代烯烃构型在卤素-镁交换反应前后得以保持; 而且，在交换反应条件下，一系列官能团 $(\mathrm{Cl}, \mathrm{I}, \mathrm{CN}$, $\mathrm{CO}_{2} \mathrm{Me}$ )得以兼容.

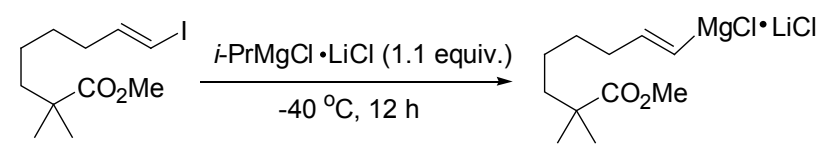

$(E: Z=99: 1)$
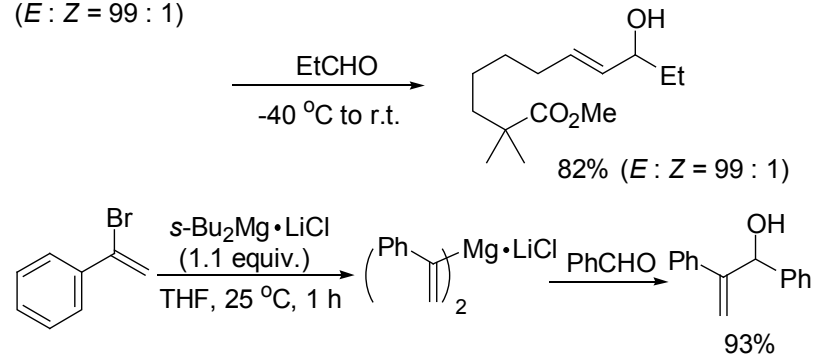

Scheme 5

尽管卤代芳烃、烯基卤代物容易和 $i$ - $\mathrm{PrMgCl} \cdot \mathrm{LiCl}$ 发生卤素-镁交换反应，但相同的反应策略用于合成烷 基镁试剂时，由于 $\mathrm{I} / \mathrm{Mg}$ 交换反应速度较慢，使得反应效 率比较低下. 2008 年, Knochel 小组 ${ }^{[14 a]}$ 报道了二异丙基 镁氯化锂 $\left(i-\mathrm{Pr}_{2} \mathrm{Mg} \cdot \mathrm{LiCl}\right)$ 和 $1,5-$ 二氯化镁戊烷氯化锂 $\left(\mathrm{ClMg}\left(\mathrm{CH}_{2}\right)_{5} \mathrm{MgCl} \cdot 2 \mathrm{LiCl}\right)$ 可以参与烷基碘代物的 $\mathrm{I} / \mathrm{Mg}$ 交换反应. 但该反应适用的烷基卤代烃比较有限. 一方 面，交换反应只适用于活性高的一级碘代物; 另一方面， 需要在 $\mathrm{C}-\mathrm{I}$ 键的 $\gamma$-位引入氮或氧等杂原子, 进而加速 $\mathrm{I} / \mathrm{Mg}$ 交换反应速度，确保交换反应的效率(Eq. 3).
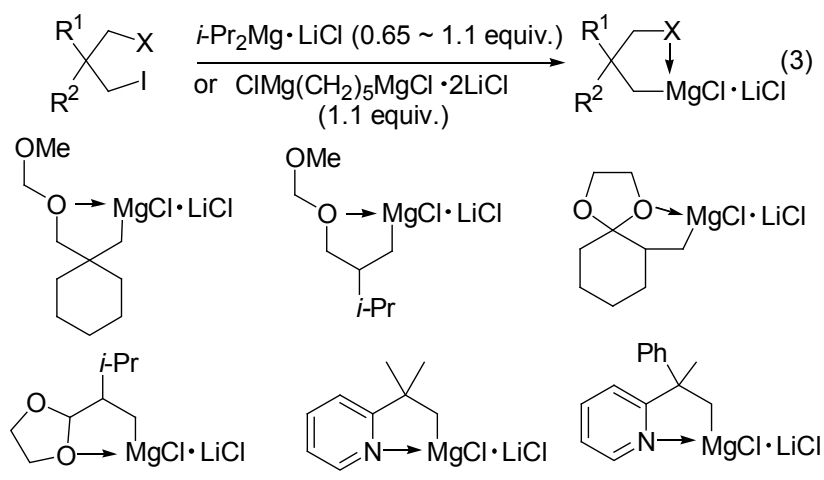

除了常用的 $\mathrm{C}-\mathrm{X}(\mathrm{X}=\mathrm{I}, \mathrm{Br}, \mathrm{Cl})$ 键可以进行卤素镁交换反应以外，芳基亚砜也可用于类似的交换反 应 $^{[15]}$. Knochel 小组 ${ }^{[16]}$ 研究发现，通过调节二芳基亚砜 中两个芳环的电性，可以解决亚砜/镁交换反应时芳环 的选择性问题(Scheme 6). 例如，在二芳基砜的其中一 个芳环的对位引入甲氧基或 $N, N-$ 二甲基时，另外一个相 对缺电子的芳环就生成相应的镁试剂. 值得一提的是, 该亚砜/镁交换反应同样适用于呋喃、苯并呋喃、噻吩等 多类杂环芳香化合物的官能团化反应. 


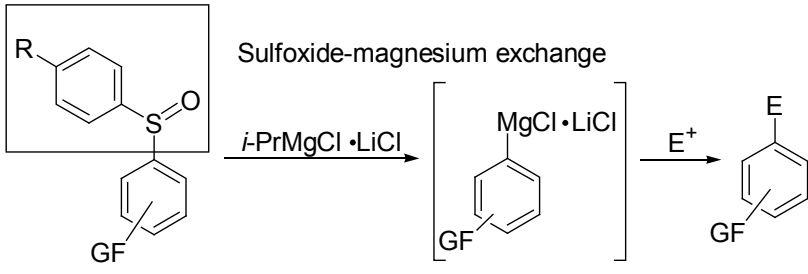

$\mathrm{R}=\mathrm{OMe}, \mathrm{NMe}_{2}$

\section{Scheme 6}

在多卤代芳烃进行卤素-镁的交换反应中, 如何实 现高选择性的卤素-镁交换反应一直是该类反应研究的 难点 ${ }^{[11,17]}$. 以异丙基氯化镁氯化锂为交换试剂时, 可以 在 2,3-二溴喹啉的 3 位和 2,4-二溴喹啉的 4 位分别进行 高选择性的 $\mathrm{Br} / \mathrm{Mg}$ 交换反应. 然而, 当进行 2,3,4-三溴 喹啉和 3,4-二溴喹啉的 $\mathrm{Br} / \mathrm{Mg}$ 交换反应时, 不同位置将 发生竞争性的 $\mathrm{Br} / \mathrm{Mg}$ 交换反应. 为此, Knochel 小组 ${ }^{[17 b]}$ 引入了比异丙基氯化镁氯化锂位阻更大的有机镁试剂 $\left(\mathrm{MesMgBr} \cdot \mathrm{LiCl}, \mathrm{Mes}_{2} \mathrm{Mg} \cdot 2 \mathrm{LiBr}\right)$, 实现了 2,3,4-三溴喹 啉和 3,4-二溴喹啉的专一 $\mathrm{Br} / \mathrm{Mg}$ 交换反应(Scheme 7). 除了通过改变有机镁试剂的空间位阻起到调控卤素/镁 交换反应的选择性以外, 胺类作为添加剂也可以用于调 控有机镁试剂的反应活性.
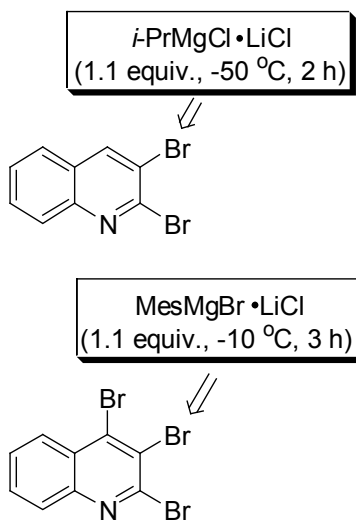

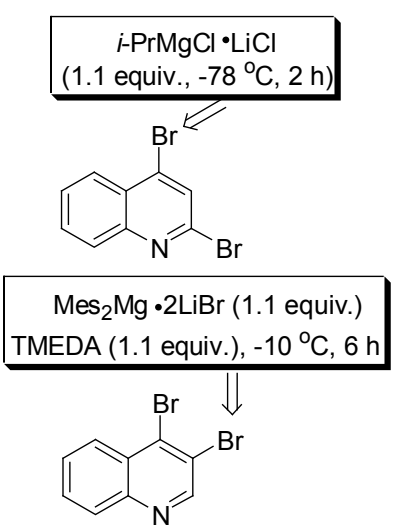

Scheme 7

通过调控格氏试剂的空间位阻，可以实现多卤代烃 的选择性卤素-镁交换反应，这样的合成策略在多卤素 取代的杂环化合物合成中得到了广泛应用 ${ }^{[17]}$. 例如以 2,3,4-三溴喹啉为起始原料, 选用 2,4,6-三甲基苯基溴化 镁氯化锂为交换试剂, 首先实现 3-位 $\mathrm{C}-\mathrm{Br}$ 键的交换反 应; 当选用 $i-\mathrm{PrMgCl} \cdot \mathrm{LiCl}$ 为交换试剂时, 4-位优先于 2位发生 $\mathrm{Br} / \mathrm{Mg}$ 交换反应; 2-位的 $\mathrm{Br} / \mathrm{Mg}$ 交换反应则选用 二(2,4,6-三甲基苯基)镁二溴化锂为交换试剂. 这样通过 三次选择性的 $\mathrm{Br} / \mathrm{Mg}$ 交换反应, 就可以合成一系列 2,3,4-三取代喹啉(Scheme 8).

\section{$1.2 \mathrm{LiCl}$ 在胺基镁参与的 $\mathrm{C}-\mathrm{H}$ 键镁化反应中的应用}

烷基锂和胺基锂是 $\mathrm{C}-\mathrm{H}$ 键金属化反应常用的有机
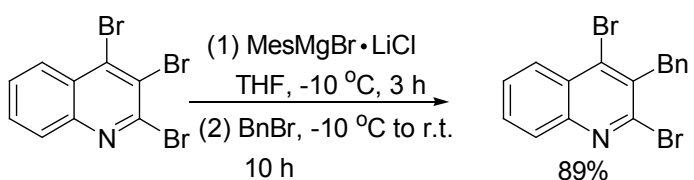

(1) $i-\mathrm{PrMgCl} \cdot \mathrm{LiCl}$ THF, $-50^{\circ} \mathrm{C}, 12 \mathrm{~h}$ (2) $\mathrm{PhSO}_{2} \mathrm{SMe}$ $-50{ }^{\circ} \mathrm{C}$ to r.t., $12 \mathrm{~h}$

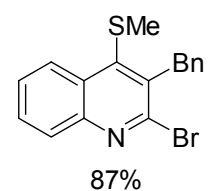

1) $\mathrm{Mes}_{2} \mathrm{Mg} \cdot 2 \mathrm{LiBr}$ $0{ }^{\circ} \mathrm{C}, 12 \mathrm{~h}$ (2) $\mathrm{Br}$ $\mathrm{CuCN} \cdot 2 \mathrm{LiCl}$ (cat.) $-20{ }^{\circ} \mathrm{C}$ to r.t., $12 \mathrm{~h}$

\section{Scheme 8}

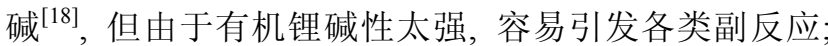
同时，胺基锂在室温下稳定性较差，因此其合成需要现 实生成，相应的锂化反应需在低温下进行，不利于在放 大反应中应用. 为此, 稳定性更好且碱性较弱的胺基镁 参与的镁化反应有望弥补胺基锂在锂化反应方面的一

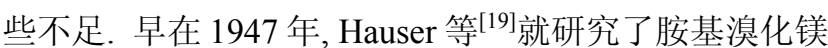
为碱进行的镁化反应(Scheme 9). 然而由于该类镁试剂 容易聚集，导致在 THF 中溶解性很差，使得镁化反应效 率低下

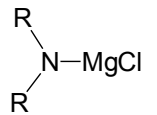<smiles>[R]NC([R])N([R])[R]</smiles><smiles>[R]N([R])[N+]([R])[R]</smiles>

\section{Scheme 9}

1989 年, Eaton 等 ${ }^{[20]}$ 报道了二(2,2',6,6'-四甲基哌啶) 镁可以用于 $\mathrm{C}-\mathrm{H}$ 键的选择性镁化反应. 和早期的 Hauser 碱相比, Eaton 等 ${ }^{[21]}$ 报道的碱在反应活性、选择 性、官能团兼容性方面都有了很大的提高，但不足的是 反应的经济性较差, 碱和亲电试剂的用量往往是镁化反 应底物的数当量(Scheme 10).

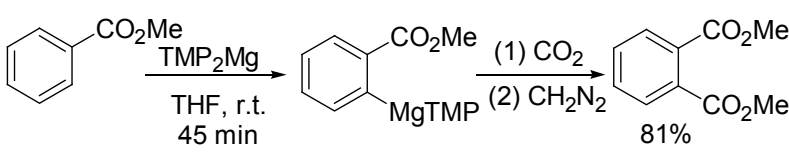

Scheme 10

2006 年, Knochel 小组 ${ }^{[6 b, 22]}$ 改进了 Hauser 碱 $\left(\mathrm{R}_{2} \mathrm{NMgBr}\right)$ ，他们将 $i-\mathrm{PrMgCl} \cdot \mathrm{LiCl}$ 和二异丙胺或 2,2',6,6'-四甲基哌啶反应制备了新型的胺基镁锂混合物, 统称为 Knochel-Hauser 碱. 和 Hauser 碱相比, 由于 $\mathrm{LiCl}$ 的存在, 胺基镁的溶解性大大提高, 从而有效地提高了 胺基镁的动力学碱性; 此外, 胺基镁的热温度性也有所 
提高, 而且参与反应时只要和底物的当量相同即可 (Scheme 11).

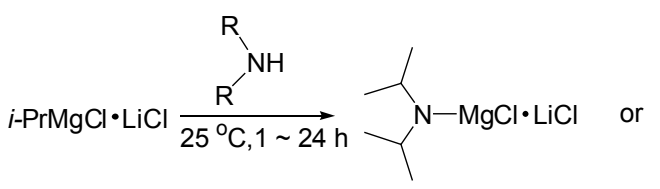

(ca. $0.6 \mathrm{~mol} / \mathrm{L}$ in THF)

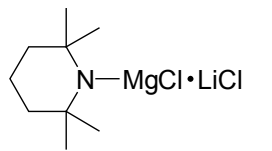

(ca. $1.2 \mathrm{~mol} / \mathrm{L}$ in THF)

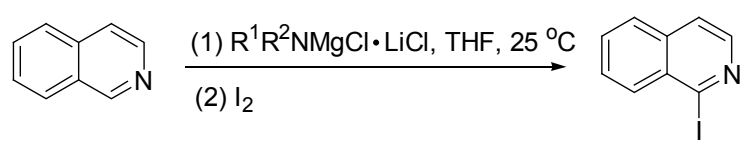

Method A: $i-\mathrm{Pr}_{2} \mathrm{NMgCl} \cdot \mathrm{LiCl}$ (2.0 equiv.), $12 \mathrm{~h}, 81 \%$;

Method B: TMPMgCl$\cdot \mathrm{LiCl}$ (1.1 equiv.), 2 h, 92\%

\section{Scheme 11}

有意思的是, 和胺基锂进行的锂化反应相比, 胺基 镁参与的镁化反应在某些反应中还表现出反应位点选 择性上的互补性. 例如, 以 LDA 为金属化试剂时, 3,5二澳吡啶选择性地在 4-位发生锂化反应 ${ }^{[23]}$; 而选用 $\mathrm{TMPMgCl} \cdot \mathrm{LiCl}$ 为碱时, 镁化反应选择性地在 2 -位进行 (Scheme 12) ${ }^{[22]}$.

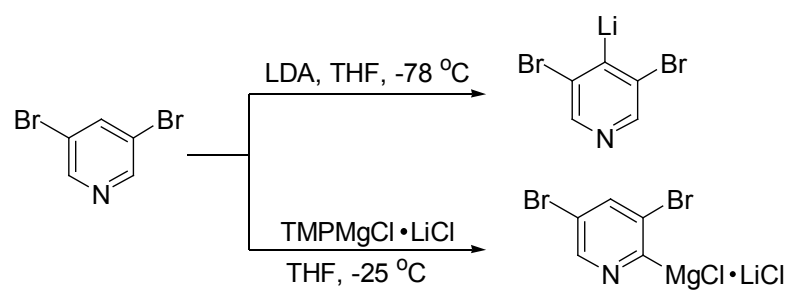

Scheme 12

尽管 $\mathrm{TMPMgCl} \cdot \mathrm{LiCl}$ 在 $\mathrm{C}-\mathrm{H}$ 键的镁化反应中表现 出很好的反应活性、底物普适性和官能团兼容性, 但对 于非活化类芳烃, 运用 $\mathrm{TMPMgCl} \cdot \mathrm{LiCl}$ 在低温下进行的 $\mathrm{C}-\mathrm{H}$ 的直接镁化反应往往不完全. 为此, Knochel 小 组 ${ }^{[24]}$ 发展了多种碱性更强的含氯化锂的二胺基镁. 例 如, 以苯甲酸叔丁酯为例, 考察的 3 个二胺基镁二氯化 锂比早期发展的 $\mathrm{TMPMgCl} \cdot \mathrm{LiCl}$ 的反应活性要高得多 (Eq. 4). 综合碱的制备、稳定性和底物普适性, 二 $\left(2,2^{\prime}, 6,6^{\prime}\right.$ - 四甲基哌啶) 镁二氯化锂 $\left(\mathrm{TMP}_{2} \mathrm{Mg} \cdot 2 \mathrm{LiCl}\right)$ 在 $\mathrm{C}-\mathrm{H}$ 键的镁化反应中得到了最为广泛的应用, 拓展了 该类反应的底物适用范围.

自 Knochel 小组 ${ }^{[6 b]}$ 改进 Hauser 碱以来, KnochelHauser 碱实现了多种类型底物的 $\mathrm{C}-\mathrm{H}$ 键镁化反应. 值 得一提的是, 在 $\mathrm{C}-\mathrm{H}$ 键的镁化反应中, 胺基镁氯化锂

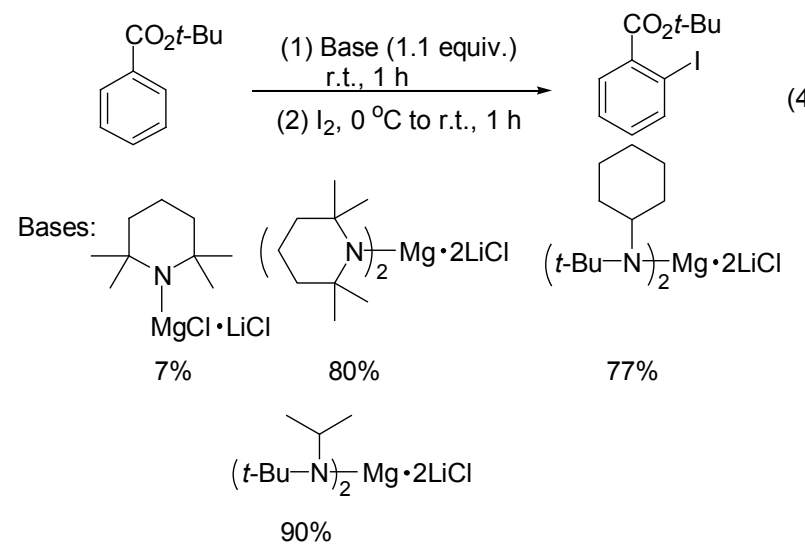

和二胺基镁二氯化锂的选用与底物具有一定的匹配性 要求 ${ }^{[25]}$. 例如, 在多卤代芳烃的选择性 $\mathrm{C}-\mathrm{H}$ 键镁化反 应中, 间二氯苯需要选用 $\mathrm{TMP}_{2} \mathrm{Mg} \cdot 2 \mathrm{LiCl}$; 而芳环更为 缺电子的均三氯苯则只需用碱性较弱的 $\mathrm{TMPMgCl} \cdot \mathrm{LiCl}$ 即可 (Scheme 13). 需要指出的是, 在选用 Knochel-Hauser 碱的反应条件下, 可有效避免 Wurtz 偶 联、卤素迁移和卤化氢消除等副反应.
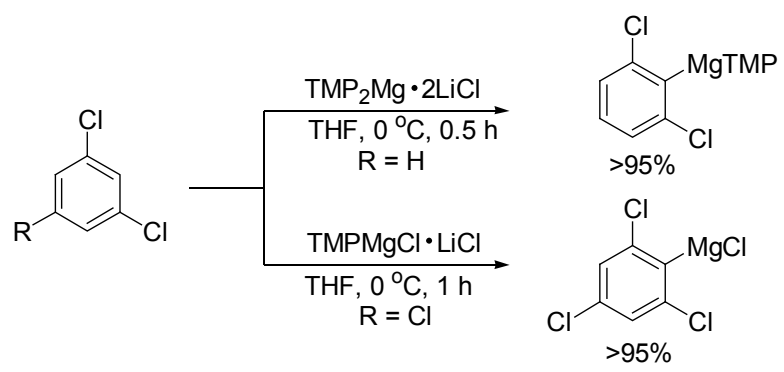

Scheme 13

在已经开展的大量关于 Knochel-Hauser 碱进行的 $\mathrm{C}-\mathrm{H}$ 键镁化反应中, 实现了选择性地对芳烃、喹啉、 异喹啉、吡啶、嘧啶、嘌呤、苯并吡嗪、呋喃、噻吩、 吡唑、 $\alpha, \beta$-不饱和烯烃、烯醇磷酸酯等类型的 $\mathrm{C}-\mathrm{H}$ 键 镁化反应, 进而实现了高区域选择性的 $\mathrm{C}-\mathrm{H}$ 键官能团 化反应 ${ }^{[6 b]}$. 相比于目前大量研究的过渡金属催化的 $\mathrm{C}-\mathrm{H}$ 键官能团化反应 ${ }^{[26]}$, 经镁化反应的官能团化反应 更具经济性、更好的选择性以及更温和的反应条件，具 备较好的应用开发前景.

定位基策略在 $\mathrm{C}-\mathrm{H}$ 键的金属化反应中得到了大量 的应用 ${ }^{[27]}$, 已发展成为一种重要的有机合成策略. Konchel 等小组 ${ }^{[28]}$ 在格氏试剂合成中也应用了邻位定位 基策略，使得格氏试剂得以在定位基的诱导下定向合 成. 例如, $N, N, N^{\prime}, N^{\prime}$-四甲基磷二酰胺基可以作为一类高 效的邻位定位基, 使 $\mathrm{C}-\mathrm{H}$ 键的镁化反应专一地在定位 基的邻位进行 ${ }^{[28 a]}$. 当定位基在芳环取代基的间位时, 由于定位基效应和位阻效应的共同作用, 镁化反应在取 代基的对位进行; 当定位基和芳环取代基处于对位时, 镁化反应则选择性地发生在取代基的间位(Scheme 14). 


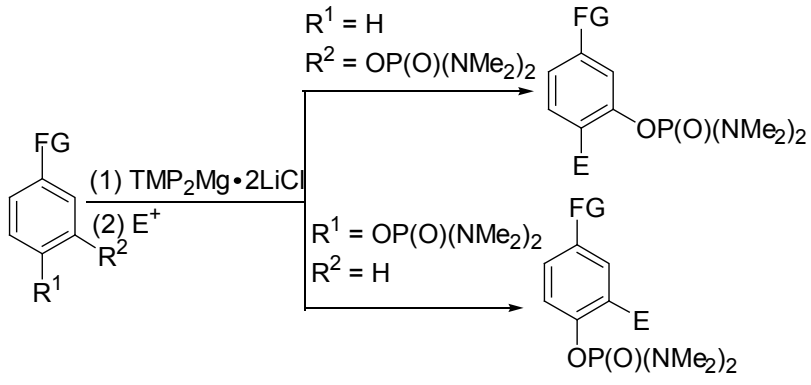

Scheme 14

由于定位基策略在镁化反应中的成功应用, 芳烃中 的 $\mathrm{C}-\mathrm{H}$ 键就可以按次序实现各类官能团化反应. 例如, 以 4-氯苯基磷二酰胺为底物时, 经 $\mathrm{C}-\mathrm{H}$ 键的镁化反应 后, 生成的有机镁试剂和 $\mathrm{ZnCl}_{2}$ 溶液发生转金属化反应 生成相应的锌试剂, 然后在 Pd 催化下和对甲氧基碘苯 进行 Negishi 偶联, 从而完成芳基氯间位的官能团化. 利用相同的反应策略，可以进一步进行芳基氯剩余间位 的官能团化反应. 值得一提的是, 芳基磷二酰胺可以在 微波反应条件下, 在甲酸、乙醇与水的混合溶液中进行 脱保护基反应, 得到多取代酚; 如若将酚转化成全氟磺 酸酯, 则可以作为下一步各类转化反应的前体(Scheme $15)$.<smiles>CN(C)P(=O)(O)Oc1ccc(Cl)cc1</smiles>
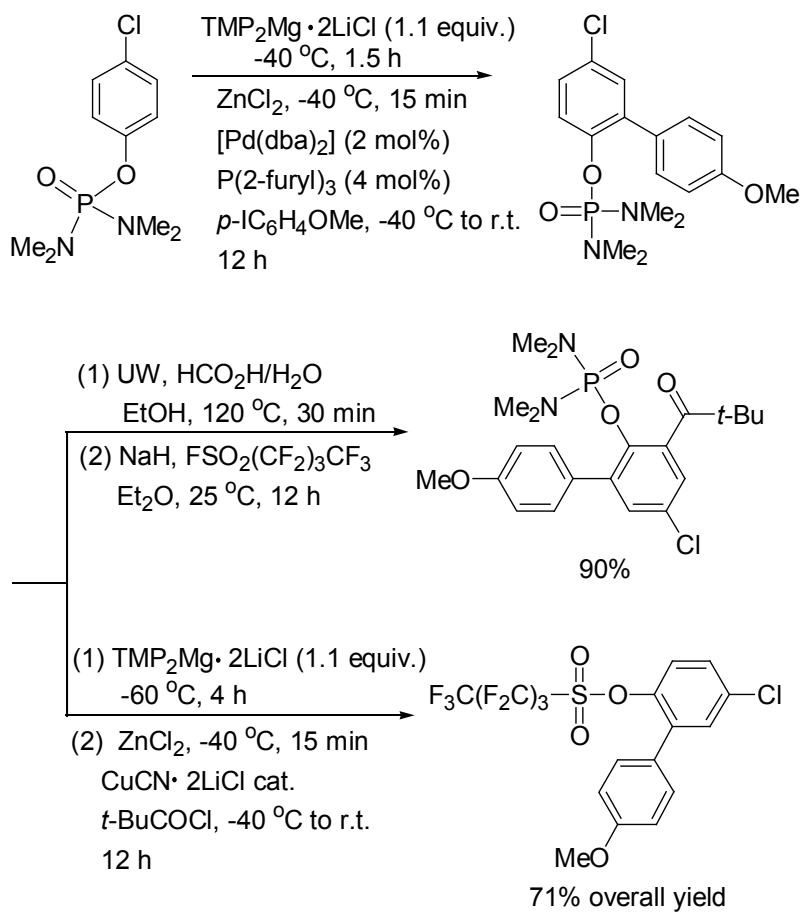

\section{Scheme 15}

除了以 $N, N, N^{\prime}, N^{\prime}$-四甲基磷二酰胺基为邻位定位基 外, 芳基亚砜在 $\mathrm{C}-\mathrm{H}$ 键的镁化反应中也可以用作邻位 定位基 ${ }^{[16]}$. 值得一提的是, 二芳基亚砜通过邻位定位效 应, 可以选择性地在其中一个芳环上实现亚砜的邻位镁
化反应; 此外, 通过亚砜-镁交换反应，就能实现亚砜原 位的官能化反应(Scheme 16). 最近, Knochel 小组以亚 砜的邻位定位效应和亚砜-镁交换反应为主要合成策略, 发展出了反应条件温和、通用性好的多官能化 7-氮杂吲 哚 ${ }^{[29 a]}$ 和咪唑衍生物 ${ }^{[29 b]}$ 的合成方法.<smiles>O=S(c1ccc(F)cc1)c1ccc(Cl)cc1</smiles>

(1) TMPMgCl$\cdot \mathrm{LiCl}$

(2) $E^{1}$

$\mathrm{R}=\mathrm{OMe}, \mathrm{NMe}_{2}$
Scheme 16

除运用定位基的策略外，路易斯酸的引入也可以实 现对吡啶和喹啉环的选择性镁化反应 ${ }^{[30]}$. 例如, 在没有 $\mathrm{BF}_{3} \cdot \mathrm{OEt}_{2}$ 存在下, 2-苯基吡啶进行的是 2 -位镁化反应; 而当反应体系中加入 $\mathrm{BF}_{3} \cdot \mathrm{OEt}_{2}$ 时, 镁化反应选择性地在 6-位进行 (Scheme 17). 同时需要指出的是, 路易斯酸的 引入不仅改变了反应的位点，同时也使相应的镁化反应 更容易进行.

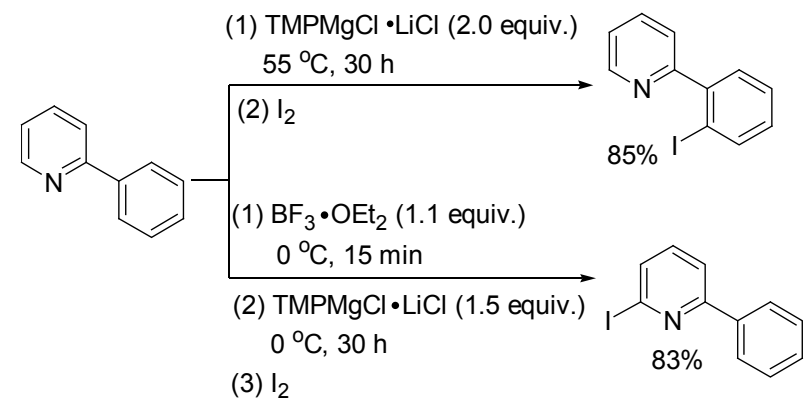

\section{Scheme 17}

在 $\mathrm{BF}_{3} \cdot \mathrm{OEt}_{2}$ 存在下，除吡啶外，喹啉、嘧啶、吡嗪 等含氮杂环也可以实现选择性的镁化反应 ${ }^{[31]}$. 特别值 得一提的是, 结构复杂的奎宁也能够选择性地在 2-位和 3-位进行镁化反应 ${ }^{[31 a]}$. 当在 $\mathrm{BF}_{3} \cdot \mathrm{OEt}_{2}$ 存在下, 用 $\mathrm{TMPMgCl} \cdot \mathrm{LiCl}$ 进行镁化反应时，由于胺基的定位效应， 反应专一地发生在喹啉环的 3-位; 而当奎宁 9-位的羟基 用大位阻的叔丁基二甲基硅基进行保护时，相应的镁化 
反应则发生在喹啉环的 2-位(Scheme 18). 从反应时间和 效率上来看, 3-位的镁化反应比 2-位要容易得多.

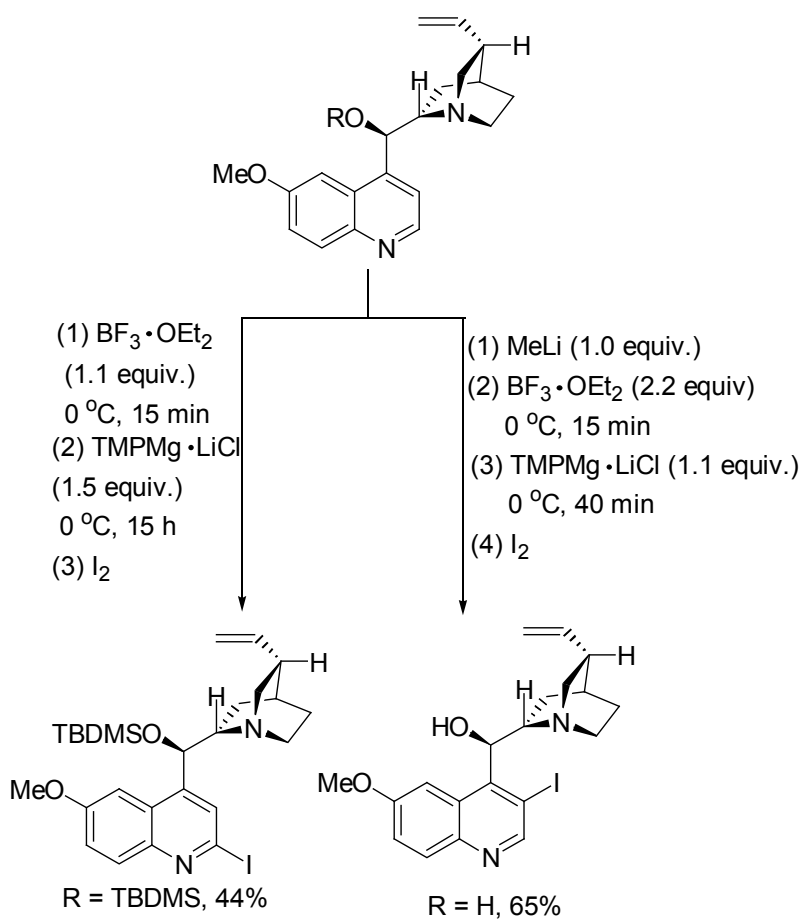

Scheme 18

Knochel-Hauser 碱除了可以实现芳环和芳香杂环的 $\mathrm{C}-\mathrm{H}$ 键镁化反应以外, 也可以对丙烯腈、丙烯酸酯、 环状烯醇磷酸酯的不饱和 $\mathrm{C}-\mathrm{H}$ 键进行选择性的镁化反 应 ${ }^{[32]}$. 例如, 以 $\mathrm{TMP}_{2} \mathrm{Mg} \cdot 2 \mathrm{LiCl}$ 为碱时, 环成烯醇磷酸 二乙酯的不饱和 $\mathrm{C}-\mathrm{H}$ 键就能发生镁化反应(Scheme 19).

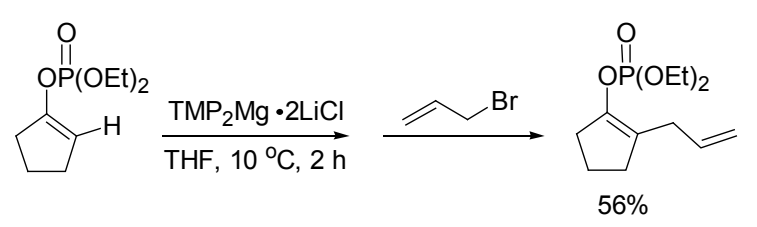

Scheme 19

Knochel-Hauser 碱除了应用于上述芳烃、烯烃等 $\mathrm{sp}^{2}$ 杂化碳的 $\mathrm{C}-\mathrm{H}$ 键镁化反应以外, 芳香杂环的茮位也可 以实现类似反应 ${ }^{[33]}$. 例如，1,2-二甲基咪唑在 1.5 equiv. 的 TMPMg$\cdot \mathrm{LiCl}$ 作用下, 即可选择性实现茮位氢的镁化 反应, 然后与 $\mathrm{ZnCl}_{2}$ 发生转金属化反应, 用于下一步 Negishi 偶联反应(Scheme 20). 但相比于 Knochel-Hauser 碱在大量 $\mathrm{sp}^{2}$ 杂化碳的 $\mathrm{C}-\mathrm{H}$ 键镁化反应中的成功应用, 烷烃类 $\mathrm{C}_{\mathrm{sp}} 3-\mathrm{H}$ 键镁化反应则还有许多工作需要开展.

\section{$1.3 \mathrm{LiCl}$ 在金属镁和有机卤化物的直接氧化插入反应 中的应用}

金属镁和有机卤化物反应是制备格氏试剂的经典 方法. 2008 年, Konchel 小组的研究表明: $\mathrm{LiCl}$ 除了对卤
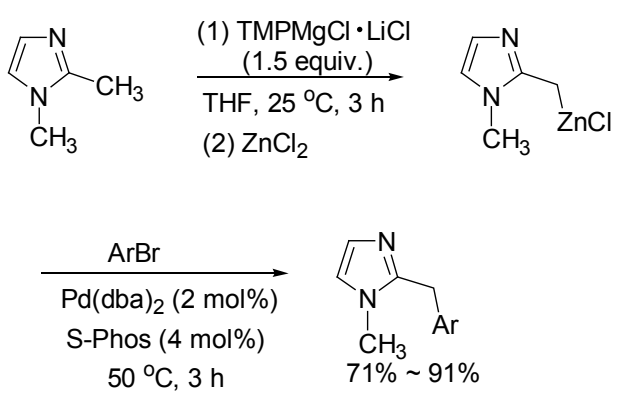

Scheme 20

素/镁交换反应、 $\mathrm{C}-\mathrm{H}$ 键的镁化反应速度有促进作用外, $\mathrm{LiCl}$ 同样可以加速 $\mathrm{Mg}$ 对卤代芳烃的氧化插入反应，同 时对格氏试剂具有一定的稳定化作用 ${ }^{[34]}$. 以2-卤代苯甲 腈为例, 没有 $\mathrm{LiCl}$ 存在下, 将卤代芳烃完全消耗完需 5 小时; 而在 1.25 equiv. $\mathrm{LiCl}$ 的存在下, 30 45 min 即可 高产率地得到目标格氏试剂(Scheme 21).

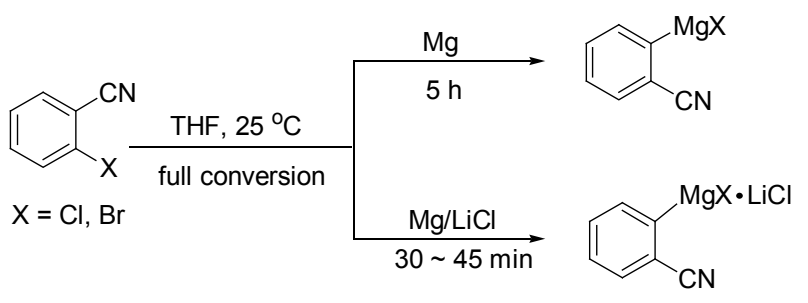

Scheme 21

由于 $\mathrm{Cl} / \mathrm{Mg}$ 交换反应速度很慢, 因此在卤素-镁交 换反应中, 常以澳代或碘代烃作为底物; 而在氯化锂存 在下, 金属镁却可以和部分氯代烃发生直接氧化插入反 应. 例如, 当在噻吩的 3,4-位引入吸电子的酯基时, 2,5位的 $\mathrm{C}-\mathrm{Cl}$ 键也可以在低温下 $\left(-50^{\circ} \mathrm{C}\right)$ 和镁进行直接的 氧化插入反应. 有意思的是, 当控制性地使用 2.5 equiv. 金属镁和 1.25 equiv. 氯化锂时，可以和其中的一个 $\mathrm{C}-\mathrm{Cl}$ 键发生氧化插入反应, 实现该位置的化学转化. 随后，应用相同的策略可以将剩余的 $\mathrm{C}-\mathrm{Cl}$ 键进行官能 团转化(Scheme 22).

此外，卤素-镁交换反应策略在烷基格氏试剂的合 成中有较多的底物限制. 而在 $\mathrm{LiCl}$ 促进下, 烷基格氏试 剂可以通过金属镁和伯澳代烷烃及仲溴代烷烃的氧化 插入反应加以合成 ${ }^{[35]}$. 例如，在烷基锌试剂的合成中, 其中一种方法就是通过格氏试剂与 $\mathrm{ZnCl}_{2}$ 发生金属交换 反应制得有机锌试剂(Eq. 5). 反应除了较高的效率和良 好的官能团兼容性以外, 反应条件也很温和, 这些都得 益于在 $\mathrm{LiCl}$ 促进条件下, 烷基格氏试剂较容易制备.

在氯化锂促进的直接氧化插入合成格氏试剂的反 应中，通过提高金属镁和氯化锂的使用量，可以方便地 实现二卤代芳烃的二次氧化插入反应，从而 “一锅法” 


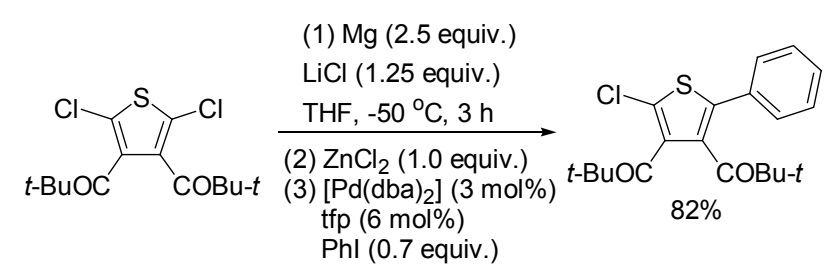

(1) $\mathrm{Mg}(2.5$ equiv.)
$\mathrm{LiCl}(1.25$ equiv. $)$
$\mathrm{THF},-50^{\circ} \mathrm{C}, 3 \mathrm{~h}$

Scheme 22

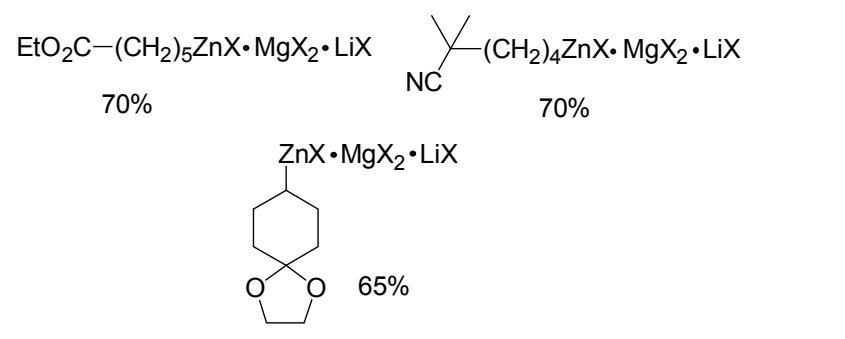

实现双格氏试剂的合成 ${ }^{[34]}$. 例如，当将金属镁试剂提高 到 10 equiv., 氯化锂用量为 5 equiv. 时, 1,4-二溴-2,5-二 甲氧基苯就可以转变成双格氏试剂; 通过后续反应条件 的控制, 实现双格氏试剂的不同转化反应(Scheme 23).
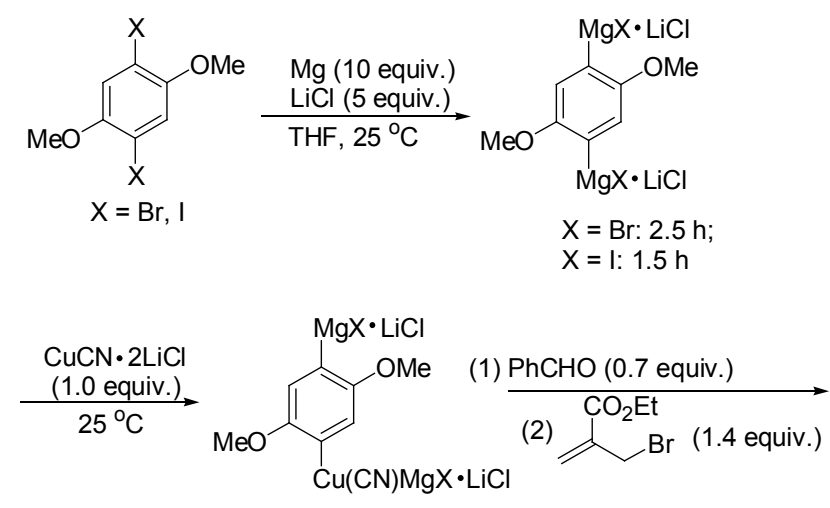<smiles>C=C(Cc1cc(OC)c(C(O)c2ccccc2)cc1OC)OCC</smiles>

Scheme 23
和卤素-镁交换反应相比，金属镁和卤代烃的直接 氧化插入法操作更简单、原子经济性也更好，同时具有 更好的官能团兼容性 ${ }^{[34]}$. 例如, 在卤素一镁交换反应中, 交换试剂 $(i-\mathrm{PrMgCl} \cdot \mathrm{LiCl})$ 具有亲核反应性能, 容易和 卤代芳烃上的羧酸酯基发生反应. 而在直接氧化插入法 中, 生成的不稳定镁试剂可以在 $\mathrm{ZnCl}_{2}$ 存在下现时转化 成活性更弱的金属锌试剂, 由此便可以解决在格氏试剂 合成中羧酸酯取代基的兼容性问题(Scheme 24).

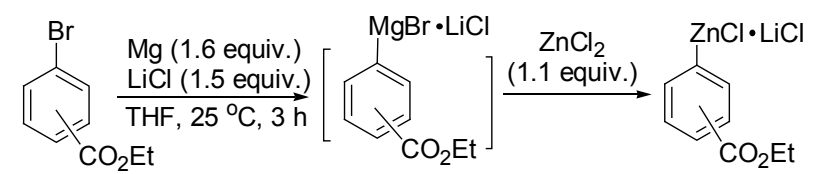

\section{Scheme 24}

\section{$1.4 \mathrm{LiCl}$ 的作用机理}

在过去的近 10 年时间里, 围绕 $\mathrm{LiCl}$ 在含多官能团 格氏试剂的合成与应用的工作层出不穷，但有关 $\mathrm{LiCl}$ 在格氏反应中的作用机制，则报道很少. 在卤素-镁交 换反应中, 由于 $\mathrm{LiCl}$ 的存在被认为可以破坏格氏交换 试剂 $(i-\mathrm{PrMgCl})$ 的聚集, 从而加快格氏交换反应 (Scheme 25). 在镁和卤代烃的直接氧化插入反应中, $\mathrm{LiCl}$ 的存在可以增加生成格氏试剂的溶解度，从而保持 镁金属表面的光洁度，确保氧化插入反应的持续进行. 在胺基镁参与的 $\mathrm{C}-\mathrm{H}$ 键镁化反应中, $\mathrm{LiCl}$ 络合的胺基 镁具有比传统 Hauser 碱更好的溶解性和动力学碱性, 从而使得 Knochel-Hauser 碱具有更好的反应活性.

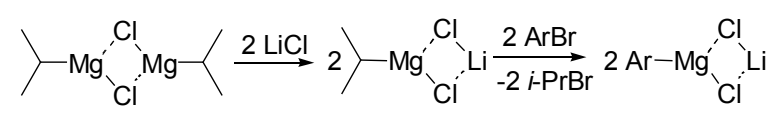

\section{Scheme 25}

综合上述三类格氏试剂合成方法的综述, $\mathrm{LiCl}$ 的引 入主要解决了格氏试剂合成中的如下三个问题: (1)解决 了绝大多数官能团在有机镁试剂合成中的兼容性问题, 极大拓展了格氏试剂的应用范围; (2)实现了反应位置可 控的高选择性有机镁试剂的合成; (3)通过有机镁试剂和 其它金属盐的转金属反应，解决了其它多官能团化有机 金属试剂的合成问题．同时，将三种有机镁合成方法相 互组合时, 根据反应物的要求, 可在同一芳环上连续且 控制性地进行格氏反应. 例如以 3-溴喹啉为起始原料, Knochel-Hauser 碱可以选择性地在 2-位进行镁化反应; 运用异丙基氯化镁氯化锂交换试剂，可以在 2,3-二澳喹 啉的 3-位选择性的发生 $\mathrm{Br} / \mathrm{Mg}$ 交换反应; 2-澳喹啉-3-羒 酸乙酯可以用 Knochel-Hauser 碱进行 4-位的 $\mathrm{C}-\mathrm{H}$ 键镁 化反应，从而选择性地先后对 2-, 3-, 4-位进行官能团转 化反应(Scheme 26). 


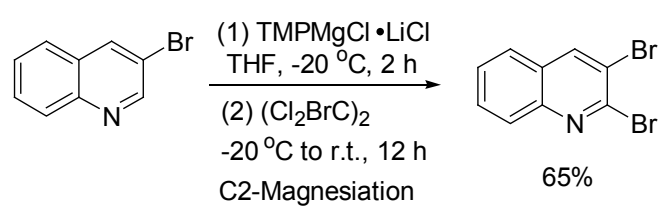

(1) $i$-PrMgCl $\bullet \mathrm{LiCl}$ $\underset{\text { (2) } \mathrm{NCCO}_{2} \mathrm{Et}}{\stackrel{\mathrm{THF}}{\mathrm{E}}-50{ }^{\circ} \mathrm{C}, 2 \mathrm{~h}}$ $-50{ }^{\circ} \mathrm{C}$ to r.t., $5 \mathrm{~h}$ C3-Magnesiation<smiles>O=C(O)c1cc2ccccc2nc1Br</smiles>
(1) $\mathrm{TMPMgCl} \cdot \mathrm{LiCl}$ (2) $\mathrm{CuCN} \cdot 2 \mathrm{LiCl}, \mathrm{THF}$ $-40^{\circ} \mathrm{C}, 10 \mathrm{~min}$ (3) $t$-BuCOCl $-40{ }^{\circ} \mathrm{C}$ to r.t., $6 \mathrm{~h}$ C4-Magnesiation<smiles>CCOC(=O)c1c(Br)nc2ccccc2c1C(=O)OCC</smiles>

\section{Scheme 26}

最近, Knochel 小组 ${ }^{[29,36]}$ 从简单的杂环化合物出发, 以 $\mathrm{LiCl}$ 参与的格氏试剂合成方法为主要合成策略, 实 现对 7-氮杂吲哚、咪唑、噁唑、吡唑、噻吩、艮嘧啶、 吡嗪等杂环化合物的多官能团化反应. 例如, 在合成多 取代 7-氮杂吲哚衍生物时, 总共在 2-, 3-, 5-, 6-位四个位 点进行了四次基于格氏反应的官能团化反应(Scheme $27)^{[29 a]}$. 这种基于 $\mathrm{LiCl}$ 促进的格氏反应的杂环官能团化 反应, 在杂环药物活性分子的修饰及其分子库的建立研 究方面具有很好的应用前景.

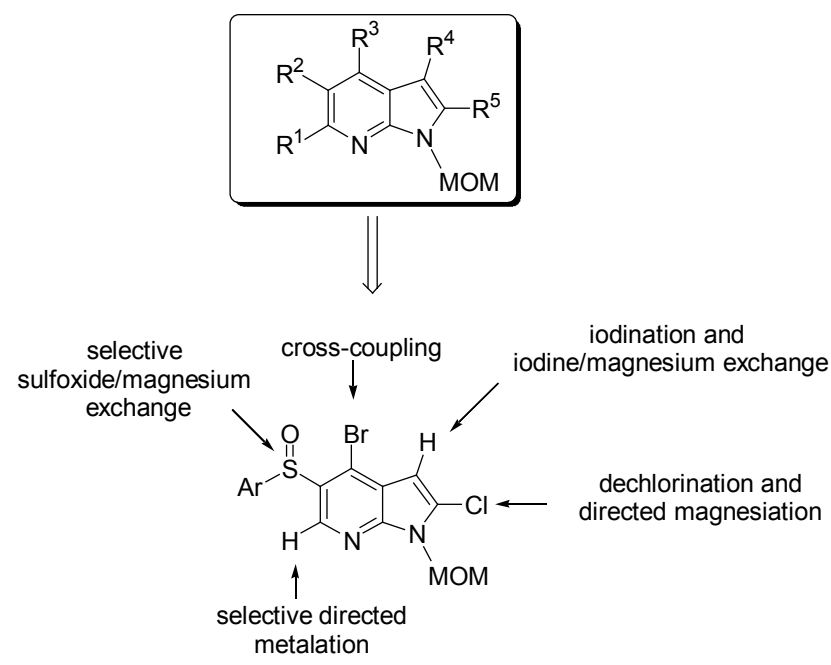

Scheme 27

\section{Knochel 型格氏试剂的应用}

\section{1 格氏试剂和各类亲电试剂反应}

格氏试剂与亲电试剂反应是格氏试剂最基本的反 应特性, Knochel 小组和其他小组在这方面进行了大量 研究, 发展出了很多基于格氏试剂官能团化的合成方
法. 例如，含多官能团的格氏试剂可以与酰氯、醛、酮、 二氧化碳、亚胺、异氰酸酯、嶰酸酯、卤代烃、三烷基 氯硅烷等各类亲电试剂反应 ${ }^{[6,17 a, 22,37]}$ (Scheme 28).

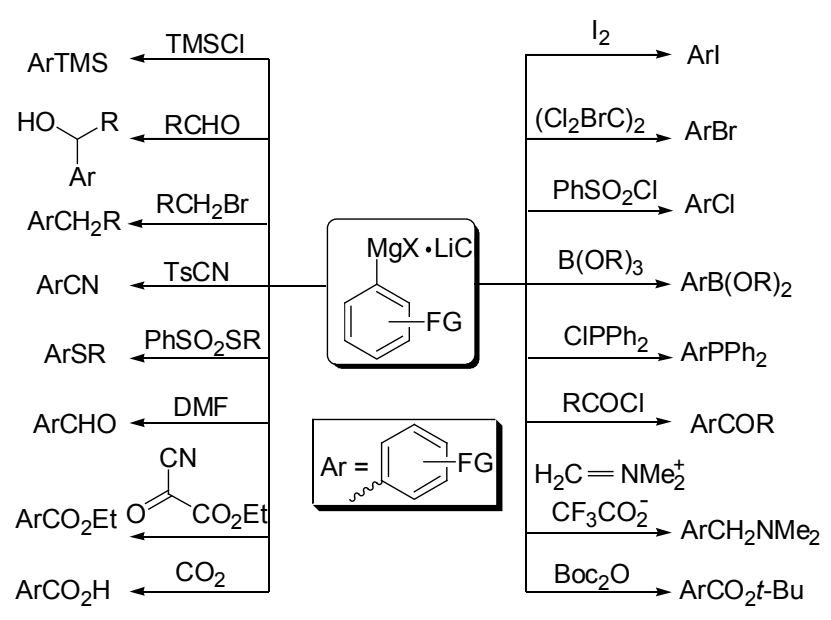

Scheme 28

特别值得一提的是, Knochel 小组 ${ }^{[38]}$ 以 $N$-氟代双苯 磺酰胺为氟化试剂, 发展了格氏试剂的氟化反应, 并可 以在实验室进行较大规模(15 20 mmol)地合成含各类 官能团的含氟芳烃、氟代杂环芳烃. 此外, Beller 小组 ${ }^{[39]}$ 也进行了类似的氟化反应研究(Eq. 6). 他们以 1-氟-2,4, 6-三甲基吡啶四氟化硼为氟化试剂, 以正庚烷或 1-甲氧 基全氟丁烷为溶剂，以中等到优秀的产率合成了各类氟 代芳烃.

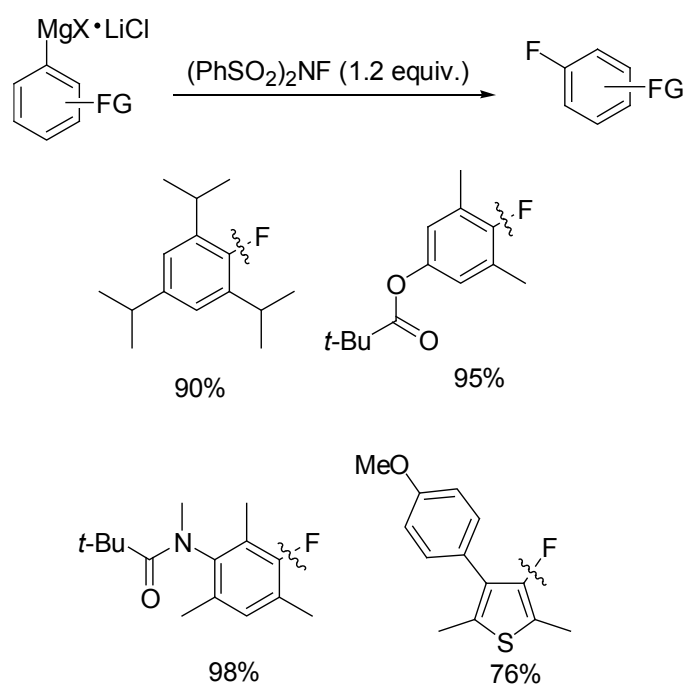

以格氏试剂为中间体是制备有机嗍酸及其衍生物 最为常用合成方法之一 ${ }^{[37 e, 40]}$. 有趣的是, Knochel 小 组 ${ }^{[41]}$ 研究发现, 卤代芳烃、镁、氯化锂、三正丁基硼酸 酯在共存情况下生成的芳基嗍镁化合物无需分离, 便可 以与各类亲电偶联体(芳基氯代物、芳基溴代物、对甲 苯磺酸芳基酯、三氟甲磺酸芳基酯和全氟丁基磺酸芳基 
酯) 在 Pd 催化条件下进行类 Suzuki-Miyaura 偶联反应 (Scheme 29). 通过控制三正丁基硼酸酯的用量, 以优秀 的产率( $>85 \%$ )合成出单芳基或二芳基硼镁化合物.

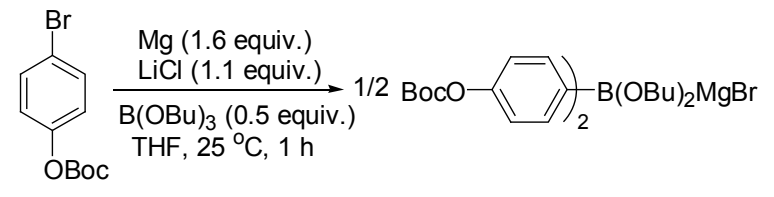

1.0 equiv.
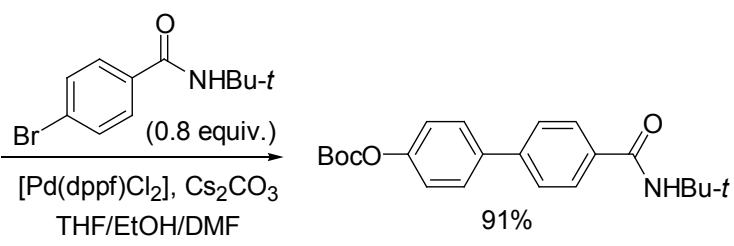

$65^{\circ} \mathrm{C}, 3 \mathrm{~h}$

Scheme 29

\section{2 格氏试剂在 C- $\mathrm{C}$ 偶联反应中的直接应用}

芳基卤代烃及类似物和有机金属试剂及类似物发 生的偶联反应是构筑 $\mathrm{C}-\mathrm{C}$ 键最重要和高效的手段之 一, 如 Kumuda-Corriu 反应 ${ }^{[3]} 、$ Suzuki-Miyaura 反应 ${ }^{[42]}$ 、 Stille 反应 ${ }^{[43]}$ 、Negishi 反应 ${ }^{[4]}{ }^{\text {等 }}{ }^{[45]}$. 在偶联反应领域里, 相比于有机硼、有机锡、有机锌、有机硅等有机偶联亲 核体, 格氏试剂突出的优势在于: (1)金属镁和卤代烃在 温和的反应条件下, 碳的三种杂化态格氏试剂都比较容 易且高效地合成; (2)镁金属单质价格低廉、分子量较小
且易于操作，适合大规模生产; (3)格氏试剂反应活性高, 可参与的反应种类多, 而且偶联反应条件温和且效率较 高. 正是基于这些优势，格氏试剂参与的偶联反应得到 了广泛的研究, 研究主要围绕新型催化剂和配体的开 发. 但在格氏试剂作为亲核偶联体参与反应时，由于有 机镁试剂的高反应活性，时常和偶联体中的各类官能团 发生副反应. 因此, 在格氏试剂参与的偶联反应中, 如 何抑制副反应成为 Kumada-Corriu 等偶联反应研究的重 点和难点.

Buchwald 小组 ${ }^{[46]}$ 在 Pd 催化的 Kumada-Corriu 偶联 反应中，选用 DavePhos 或 S-Phos 等单膦配体时，各类 芳基碘代物和芳基格氏试剂可以在低温反应条件下 $\left(-20 \sim-65{ }^{\circ} \mathrm{C}\right)$ 高效偶联(Scheme 30). 应用 Knochel 的卤素/镁交换反应可以合成出含各类官能团的格氏试 剂，在Buchwald 小组发展的催化体系下，便可以高效地 合成出含各类官能团的联苯类化合物.

在 Kumada-Corriu 偶联反应的催化体系中, 选用比 $\mathrm{Pd}$ 催化体系更为经济且反应活性相当的新型催化体系 是该类偶联反应中的重要研究方向 ${ }^{[47]}$. 最近, Knochel 小组 ${ }^{[48}$ 报道了在 $\mathrm{Fe}$ 或 $\mathrm{Co}$ 催化的芳基格氏试剂和氮杂卤 代芳烃的偶联反应中, 催化量的喹啉或异喹啉可以有效 地促进该类 $\mathrm{C}-\mathrm{C}$ 偶联反应(Eq. 7). 例如, 在 $\mathrm{FeBr}_{3}$ 催化 的偶联反应中，在没有异喹啉的存在下，偶联反应的产 率只有 $10 \%$; 而加入 $10 \mathrm{~mol} \%$ 的异喹啉后，反应产率可
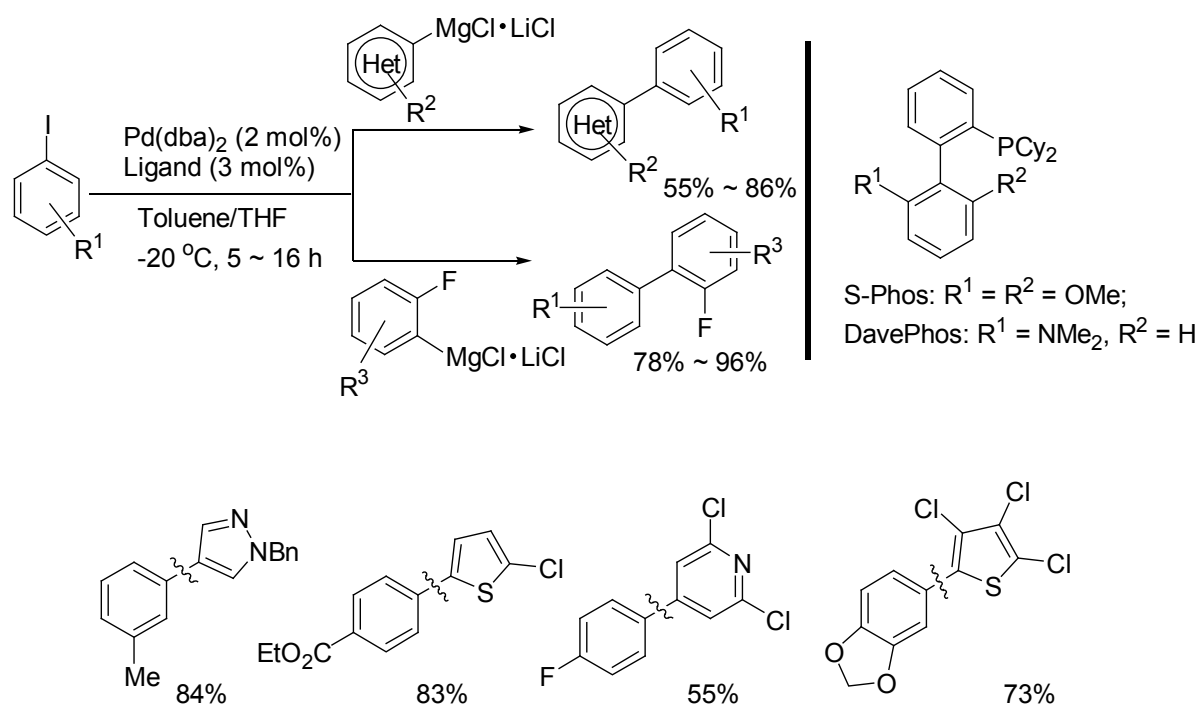<smiles>O=C(c1ccccc1)c1c(F)cccc1F</smiles>

$81 \%$<smiles>Fc1cccc(-c2ccc3c(c2)OCO3)c1F</smiles>

$93 \%$<smiles>Fc1cccc(-c2ccccc2CO[Na])c1F</smiles><smiles>Fc1cccc(-c2cccc3ccccc23)c1F</smiles>

Scheme 30 
以上升至 $71 \%$, 极大地提高了偶联效率. 同样, 在 $\mathrm{CoCl}_{2}$ 催化的同类偶联反应中, 异喹啉也可以提升偶联效率. 通过自由基钟实验, Knochel 小组认为: 在 $\mathrm{Fe}$ 或 $\mathrm{Co}$ 催化 的 $\mathrm{C}-\mathrm{C}$ 偶联反应中, 至少有部分是自由基反应历程; 而相应的 $\left[\mathrm{Pd}\left(\mathrm{PPh}_{3}\right)_{4}\right]$ 催化的偶联反应则基本观察不到经 自由基中间体反应得到的环合产物。
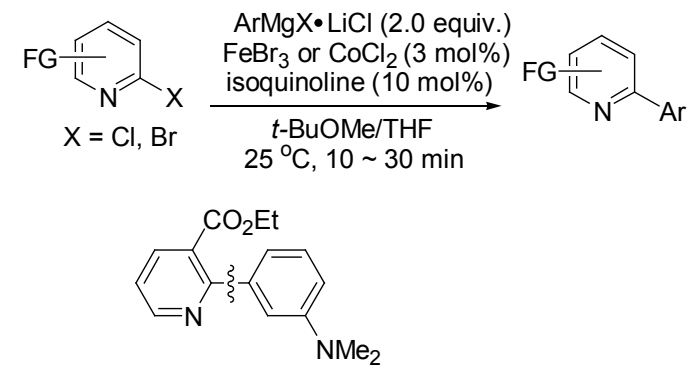

$\mathrm{FeBr}_{3}$ (3 mol\%)/isoquinoline (10 mol\%): $71 \%$; $\mathrm{FeBr}_{3}(3 \mathrm{~mol} \%): 10 \%$

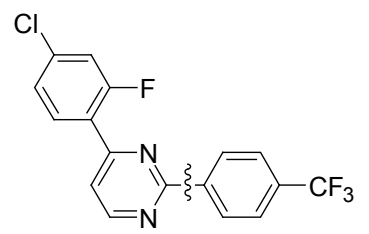

$\mathrm{CoCl}_{2}$ (3 mol\%)/isoquinoline (10 mol\%): $70 \%$; $\mathrm{CoCl}_{2}(3 \mathrm{~mol} \%): 43 \%$

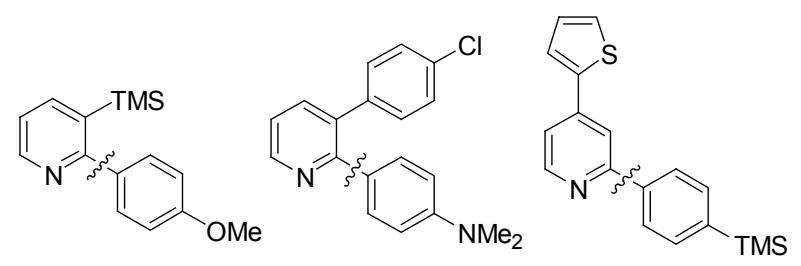

(Fe: $91 \%$; Co: $85 \%$ ) (Fe: 82\%; Co: $77 \%$ ) (Fe: $65 \%$; Co: $70 \%$ )
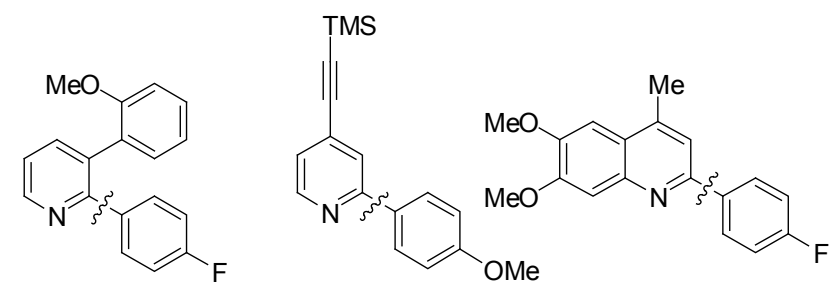

(Fe: $77 \%$; Co: $79 \%$ )

(Fe: $38 \%$; Co: $62 \%$ )

(Fe: 82\%; Co: 67\%)

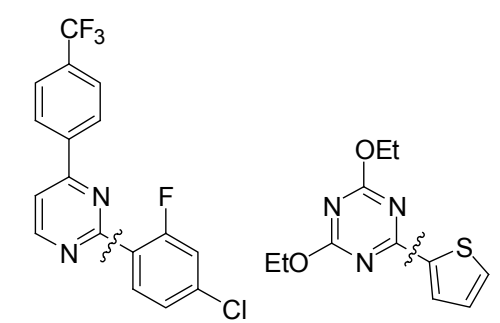

(Fe: $61 \%$; Co: $60 \%$ ) (Fe: $84 \%$; Co: $79 \%$ )

在过渡金属催化的偶联反应中, 除经典的偶联反应 历程外, 自由基反应等其它历程也是可能的反应途
径 ${ }^{[49]}$. 基于自由基反应历程的设想, Knochel 小组 ${ }^{[50]}$ 发 展出了 Pd 催化条件下, 异丙基碘促进的 Kumada-Corriu 偶联反应. 当以 3 -碘三氟甲苯和 3 -溴三氟甲苯通过卤素 一镁交换反应制得的格式试剂在相同反应条件下进行 Kumada 偶联反应时, 反应效率差别很大(Scheme 31). 通过这个对照实验可以看出，碘-镁交换反应生成的副 产物异丙基碘可以促进 Kumada-Corriu 偶联反应的进 行. Knochel 小组认为, 异丙基碘的存在有利于自由基的 引发反应，从而使得 $\mathrm{C}-\mathrm{C}$ 偶联反应有利于自由基反应 历程进行. 此外, 在 Pd 催化的 Negishi 反应中, 异丙基 碘也有类似的促进作用 ${ }^{[51]}$.

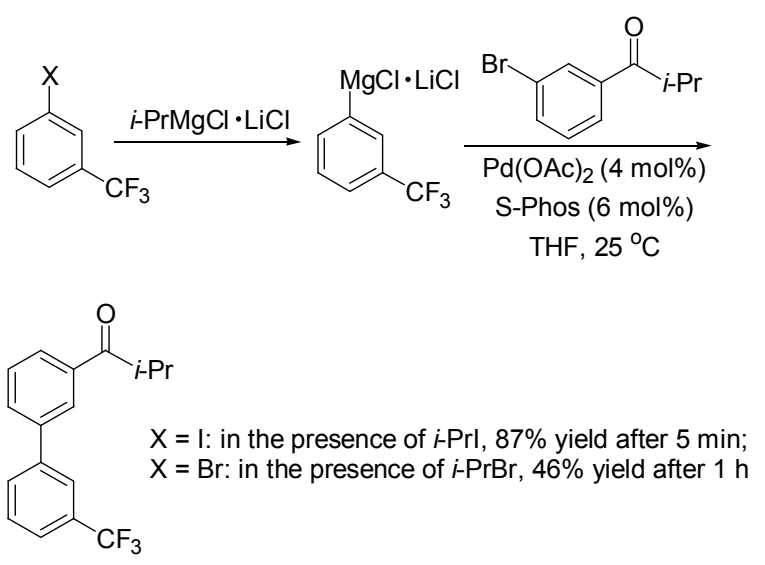

\section{Scheme 31}

在亚铜/亚磷酸三乙酯/四丁基碘化铵的催化条件下, 芐基二乙酯磷酸酯和格氏试剂在较温和的反应条件下, 可以实现高效 $\mathrm{C}-\mathrm{C}$ 偶联, 从而用于合成高官能团化的 二芳基甲烷类化合物 ${ }^{[52]}$. 例如, 5-溴-2,4-二叔丁基嘧啶 通过 $\mathrm{Br} / \mathrm{Mg}$ 交换反应制得相应格氏试剂, 然后在亚铜催 化条件下，格氏试剂和 3,4,5-三甲氧基芐基二乙酯磷酸 酯发生 $\mathrm{C}-\mathrm{C}$ 偶联反应，以 $81 \%$ 的产率得到用于合成抗 生素化合物 Trimethoprim 的中间体(Eq. 8).

格氏试剂参与的 $\mathrm{C}-\mathrm{C}$ 偶联反应中, 有交叉偶联和 自身偶联两类偶联反应. 在众多的偶联反应研究中，除 了大量过渡金属催化的偶联反应报道以外，无金属参与 下的格氏试剂偶联反应也得到了广泛关注 ${ }^{[53]}$. Konchel 小组 ${ }^{[54]}$ 利用 3,3',5,5'-四叔丁基- $4,4^{\prime}$-联苯醌为电子受体, 在非常温和的条件下，实现了格氏试剂的自身偶联反 应，从而高效合成含各类官能团的联苯、联菜、共轭二 炔烃、共轭二烯烃等偶联产物(Eq. 9). 需要指出的是, 偶 联体的电性、空间位阻对偶联反应效率影响较小. 同时 自由基钟类底物只进行自身偶联反应，没有环合产物; 而且烯烃在偶联反应前后，构型均保持一致. 作者从这 两点提出了可能的反应机理, 认为反应是单电子转移反 

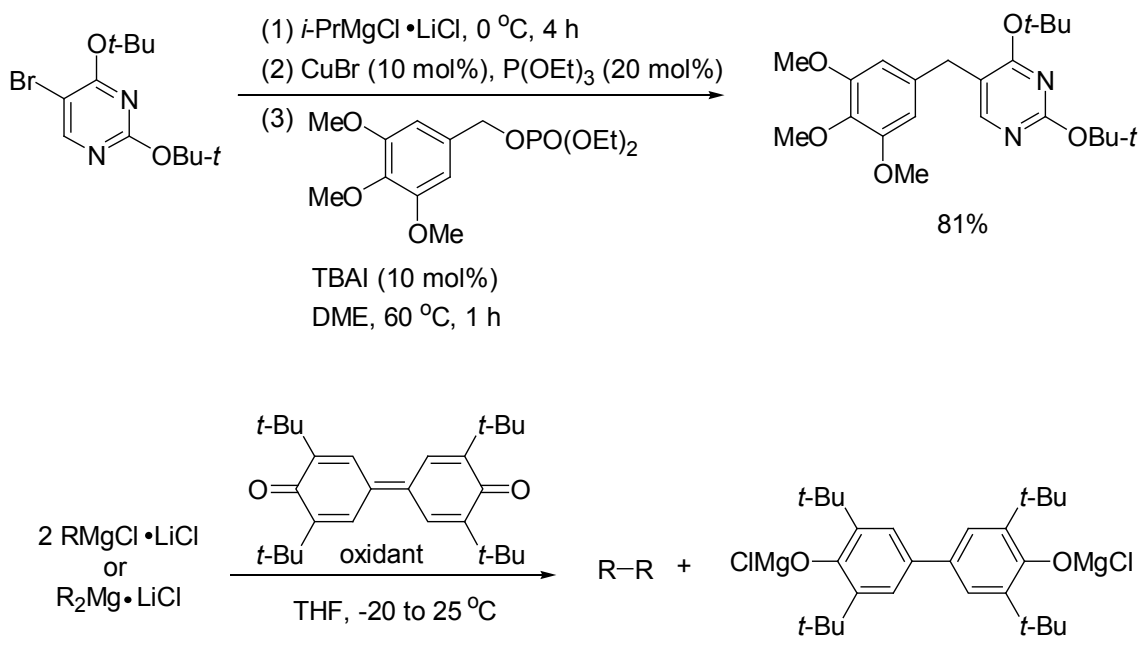
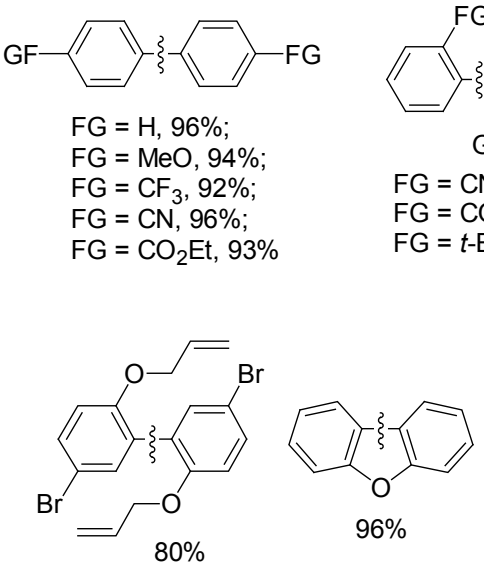

应历程. 电子受体的反应产物联二酚经过滤等简单操作 后很容易从反应体系中过滤分离; 而且在空气氧化条件 下, 联二酚可以定量地转化成联苯醌, 这样一方面提高 了反应的经济性，同时也展现了很好的放大应用前景.

\section{3 转金属反应}

相比于其它金属试剂的制备，格氏试剂的合成往往 更高效、直接. 基于这个特性, 它往往是合成其它金属 试剂的前体. 通过格氏试剂和相应金属盐的金属交换反 应，可以实现锌 ${ }^{[55]}$ 、铜 ${ }^{[17 \mathrm{c}, 56] \text { 、铟 }}{ }^{[57]}$ 等有机金属试剂的高 效合成 ${ }^{[6 a, 6 b]}$. 此外, 一些有机镁试剂在反应中由于缺乏 足够的稳定性, 从而限制了格氏试剂的进一步应用. 在 这些情况下, 往往采用转金属反应策略, 将生成的有机 镁试剂即时转化为更为稳定的有机锌、有机铜等其它有 机金属试剂，进而参与下一步目标反应.

即使在氯化锂存在下, 2-溴-1-环己烯-1-甲酸乙酯也 不能和锌进行直接的氧化插入反应, 而金属镁则可以进 行氧化插入反应得到烯基格氏试剂. 制得的格氏试剂现 时和 $\mathrm{ZnCl}_{2}$ 发生金属交换反应, 生成金属锌反应, 进而 参与各类后续反应(Eq. 10) ${ }^{[55 \mathrm{~d}]}$. 因此, 通过镁的氧化插
入反应/转金属反应的接续反应来合成各类金属试剂, 可以弥补其它金属直接氧化插入反应活性不足的缺点.
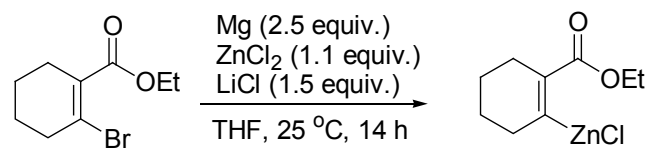

在苯并吡嗪的 2-位镁化反应中, 如果在没有 $\mathrm{ZnCl}_{2}$ 存在下, 通过 $\mathrm{I}_{2}$ 的淬灭反应可以发现，二聚物是主要的 产物，而目标的碘代物只有微量产物; 当上面的反应体 系中加入 0.5 equiv. $\mathrm{ZnCl}_{2}$ 时，目标碘代产物可以上升到 $94 \%$ ，自身偶联等副反应就可以得到抑制(Eq. 11) ${ }^{[58]}$. 从 这个例子可以看出, 通过转金属反应可以避免有机镁试 剂稳定性不足的缺点，进而控制反应途径.

除了各类烃基镁试剂能发生各类转金属反应以外, 胺基镁也可以发生类似的转金属反应，从而提供了胺基

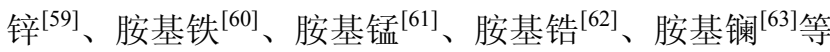
金属试剂的通用且高效的合成方法 ${ }^{[6 b]}$. 例如，当 Knochel-Hauser 碱与 $\mathrm{ZnCl}_{2}$ 反应制备胺基锌时，产率接 
近定量(Eq. 12).

\section{(1) $\mathrm{TMP}_{2} \mathrm{Mg} \cdot 2 \mathrm{LiCl}(0.55$ equiv.) $25^{\circ} \mathrm{C}, 2 \mathrm{~h}$}

(2) $\mathrm{I}_{2}, 0$ to $25^{\circ} \mathrm{C}, 1 \mathrm{~h}$<smiles>c1ccc2nc(-c3cnc4ccccc4n3)cnc2c1</smiles>
without $\mathrm{ZnCl}_{2}$ : traces $34 \%$ with $\mathrm{ZnCl}_{2}$ (0.50 equiv.): $\quad 94 \%$

traces

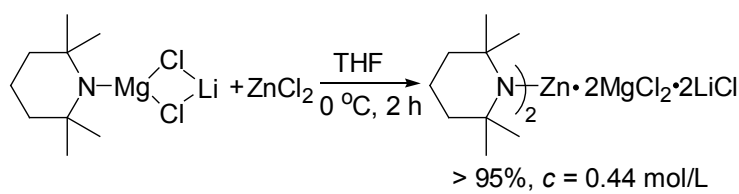

\section{4 在低聚物和聚合物合成中的应用}

低聚噻吩在有机薄膜晶体管 ${ }^{[64]}$ 、液晶材料 ${ }^{[65]}$ 、染料 敏化有机光伏电池 ${ }^{[66]}$ 等材料领域应用极为广泛，因此， 高效简便的噻吩低聚物合成方法是有机合成与材料科 学的重要研究课题之一 ${ }^{[67]}$. Mori 小组 ${ }^{[68]}$ 最近在研究 $\mathrm{Ni}$ 催化的噻吩格氏试剂的聚合反应中发现, 在 Knochel-Hauser 碱促进的 $\mathrm{C}-\mathrm{H}$ 键镁化反应中, 无论是 单体噻吩(3-己基噻吩), 还是二聚噻吩(3,4'-二己基-2,2'二噻吩), 镁化反应都专一地发生在己基的间位. 基于 这样的选择性, 生成的格氏试剂在 $\mathrm{Ni}$ 的催化作用下和 2-溴-3-己基噻吩发生 $\mathrm{C}-\mathrm{C}$ 偶联生成头-尾型低聚物. 因此, 只要反复利用 Knochel-Hauser 碱促进的 $\mathrm{C}-\mathrm{H}$ 键 镁化反应/ $\mathrm{Ni}$ 催化的 $\mathrm{C}-\mathrm{C}$ 偶联反应策略, 即能合成头尾型结构、聚合度可控的噻吩低聚物(Scheme 32).

格氏试剂除了在低聚物合成中的应用以外, 它在聚 (2,7-二烷基芴)等共轭聚合物的合成中也被普遍应用 ${ }^{[69]}$. 相比基于 Yamamoto、Suzuki、Still 等偶联反应的聚合 方法，格氏置换 (Grignard Metathesis, GRIM) 聚合具有 反应时间短和反应条件温和等优点 ${ }^{[70]}$. Yokozawa ${ }^{[71]}$ 、 McCullough ${ }^{[72]}$ 等小组应用格氏置换聚合方法合成共轭 高分子时发现, $\mathrm{LiCl}$ 的存在可以促进单体生成格氏试剂 的效率, 从而提高聚合效率. 例如, 以异丙基氯化锂作 为交换试剂时，2,7-二溴-9,9-二辛基茀交换效率很低 (5\%); 而当采用双金属的异丙基氯化镁氯化锂作为交换 试剂时，反应效率可以提升至 $85 \%$ (Scheme 33) ${ }^{[72]}$.

和大量 $\mathrm{Ni}$ 催化的格氏置换聚合反应不同的是, Studer 小组 ${ }^{[73]}$ 报道了双格氏试剂在没有过渡金属参与 条件下的氧化聚合反应. 他们以 2,2,6,6-四甲基哌啶-1氧化物(TEMPO)为氧化剂, 双格氏试剂可以在氧化条件
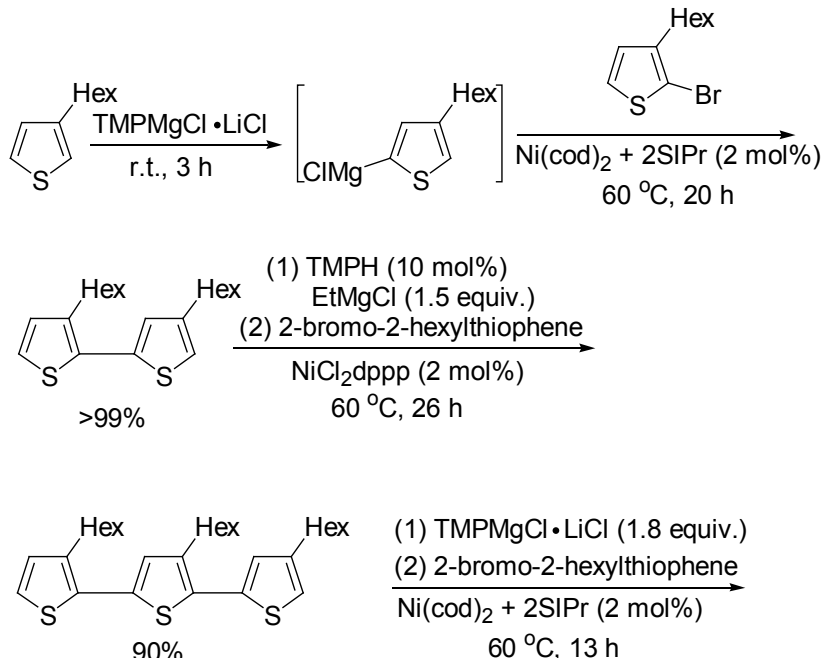

(1) $\mathrm{TMPMgCl} \cdot \mathrm{LiCl}(1.8$ equiv.) (2) 2-bromo-2-hexylthiophene $\mathrm{Ni}(\mathrm{cod})_{2}+2 \mathrm{SIPr}(2 \mathrm{~mol} \%)$ $60{ }^{\circ} \mathrm{C}, 13 \mathrm{~h}$

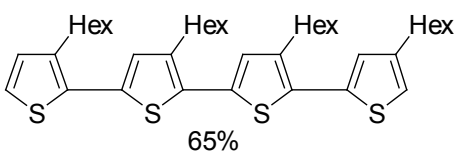

Head-to-Tail-Type Oligothiophene

Scheme 32
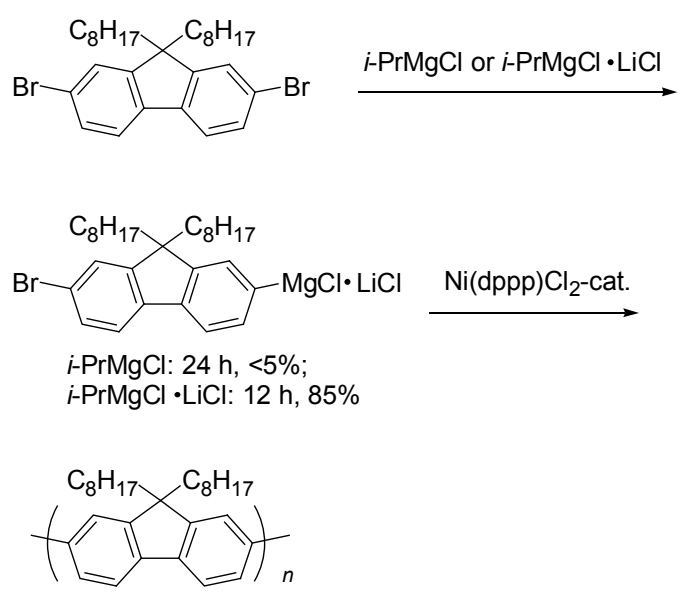

$88 \%$ conversion

$M_{\mathrm{n}}=4800 \mathrm{~g} / \mathrm{mol}, \mathrm{PDI}=1.45$

\section{Scheme 33}

下高效聚合, 得到聚(9,9-二辛基芴)类共轭高分子 (Scheme 34). 要指出的是, 在镍催化条件和氧化聚合反 应条件下，格氏试剂的合成方法都对聚合效率与质量有 较大影响.

\section{5 其它亲核反应中的应用}

格氏试剂是有机合成中最常用的碳亲核试剂之一， 含氯化锂的格氏试剂除了上述提到的可以和各类亲电 试剂反应以外, 还可以参与多类亲核取代和亲核加成反 应.

最近, Knochel 小组 ${ }^{[74]}$ 报道了在路易斯酸 $\left(\mathrm{BF}_{3} \bullet \mathrm{OEt}\right)$ 促进的吡啶环的官能团化反应，这对过渡金属催化或促 


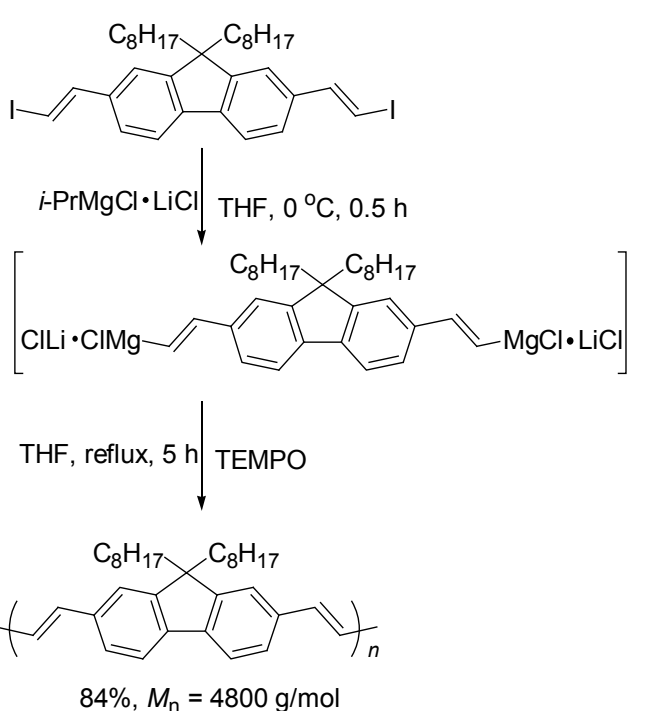

Scheme 34

进的吡啶环官能团化反应是个有利补充 ${ }^{[26 c, 75]}$. 例如, 3氯吡啶通过和 $\mathrm{BF}_{3} \cdot \mathrm{OEt}$ 络合, 降低了吡定环的电子云密 度, 从而接受格氏试剂的亲核加成反应生成 1,4-二氢吡 啶中间体. 最后, 在四氯对苯醌氧化剂的氧化条件下完 成芳构化反应，以 $89 \%$ 的产率得到 3-氯-4-异丙基吡啶 (Scheme 35).

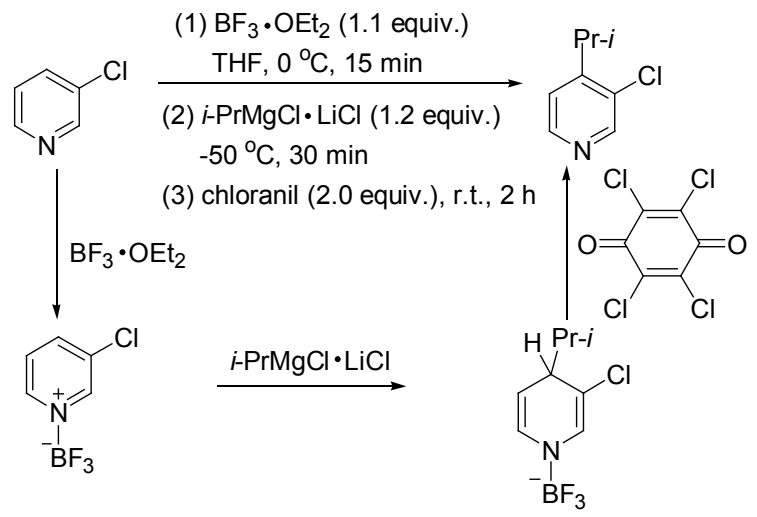

Scheme 35

在上述取代反应中, 各级烷基格氏试剂和多官能团 化的芳基格氏试剂均可以用于吡啶环的烷基化和芳基 化反应(Scheme 36). 同时, 该取代反应具有位点选择专 一性特点. 当吡啶和喹啉环的 4-位没有取代基时, 反应 专一地在 4-位进行烷基化或芳基化反应; 当 4-位有取代 基时, 取代反应则发生在 2-位, 但相应的反应效率比 4位取代反应差一些. 此外, 双格氏试剂可以和两分子吡 啶环发生取代反应。通过以上对照实验发现, 格氏试剂 的亲核反应能力与吡啶环的电性对反应速率有较大影 响.

含氯化锂的烯基格氏试剂可以对二芳基偶氮化合 物进行 $N=N$ 双键的亲核加成, 生成 Fischer 吲哚合成法<smiles>CCc1ccnc(Cl)c1</smiles><smiles>CCCCC(C)C</smiles><smiles>CC(Br)(Br)c1ccncc1C#N</smiles><smiles>Clc1cnccc1C=[W]</smiles><smiles>COc1ccc2nccc(C(C)C)c2c1</smiles><smiles>CCOC(=O)c1cnccc1OCC</smiles><smiles>N#Cc1ccnc(-c2cccs2)c1</smiles><smiles>N#CC1=C(Cl)C=C[NH+]1O</smiles><smiles>COc1cc(-c2ccncc2C#N)c(OC)cc1-c1ccncc1C</smiles>

Scheme 36

的关键中间体，烯基和芳基取代的联胺中间体在微波反 应条件下即可合成多取代的吲哚(Scheme 37 ${ }^{[76]}$.

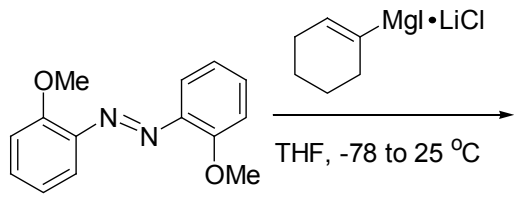<smiles>[X]c1ccccc1N(C1=CCCCC1)N(C)c1ccccc1OC</smiles>

TMSCl (1.0 equiv.)

$\operatorname{NMP}\left(20\right.$ vol\%), $125^{\circ} \mathrm{C}, 30 \mathrm{~min}$

mircrowave irradition

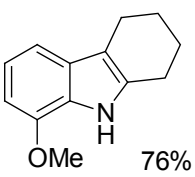

Scheme 37

除了 $\mathrm{N}=\mathrm{N}$ 双键，格氏试剂对 $\mathrm{N}=\mathrm{O}$ 双键也有亲核 加成的报道. 例如，芳香族硝基化合物可以和芳基格氏 试剂发生反应，中间产物经 $\mathrm{FeCl}_{2}$ 和 $\mathrm{NaBH}_{4}$ 还原，就可 以在温和条件下制备二芳胺 (Scheme 38) ${ }^{[77]}$. 根据设想 的反应机理，反应中包含了格氏试剂对中间体亚硝基不 饱和键的亲核加成. 由于反应条件的温和性及良好的官 能团兼容性，该方法是过渡金属催化制备二芳胺的有利 补充 $^{[78]}$.

\section{3 含 $\mathrm{LiCl}$ 格氏试剂在天然产物合成中的应用}

格氏试剂是天然产物合成中常用的 $\mathrm{C}-\mathrm{C}$ 键构筑试 剂，传统的方法由于在官能团兼容上的局限，限制了格 氏试剂在复杂天然产物合成中的应用. 随着 Knochel 小 组在格氏试剂合成上的突破，使得格氏试剂的合成条件 更为温和、合成效率和反应活性更高，从而使得格氏试 剂在复杂天然产物合成中的应用成为了可能. 


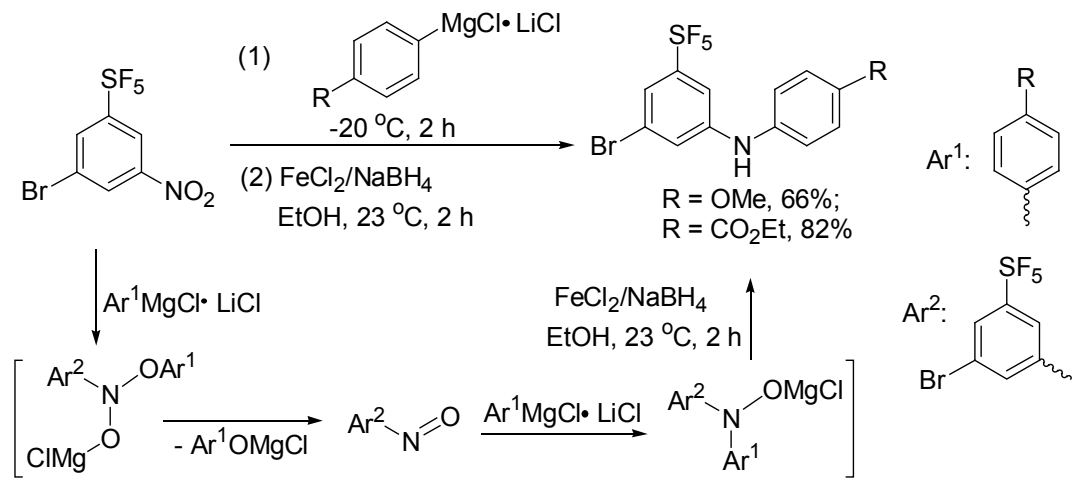

\section{Scheme 38}

2009 年, Castle 小组 ${ }^{[79]}$ 报道了四环生物碱(一)acutumine 的全合成, 其中自由基的共轭加成一烯醇离子 的羟基化串联反应是苯并环戊烷结构构建的关键策略. 在合成串联反应前体时, Castle 小组利用 $i-\mathrm{PrMgCl} \cdot \mathrm{LiCl}$ 为交换试剂, 通过 $\mathrm{I} / \mathrm{Mg}$ 交换反应制得了烯基格氏试剂, 进而和 Weinreb 酰胺反应以优秀的产率合成了目标 $\alpha, \beta$ 不饱和酮(Scheme 39).<smiles>COc1c(Cc2ccccc2)cc(N(C)OC)c(OC)c1CC(=O)N(C)OC</smiles>

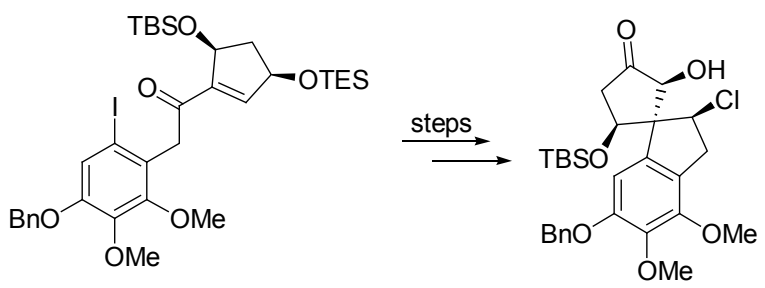

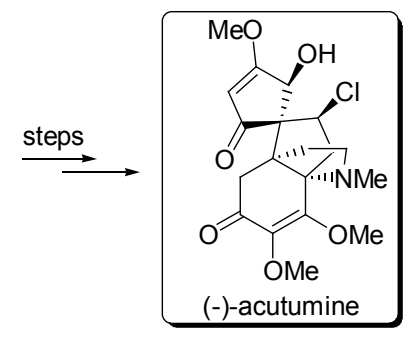

Scheme 39

2009 年, 祝介平小组 ${ }^{[80]}$ 以芳基格氏试剂对手性氮 杂环丙烷的开环反应为关键合成策略, 成功合成了具有 生物活性的四氢异喹啉生物碱家族中的若干个化合物. 例如, 在(一)-Jorumycin 全合成中, 在 $\mathrm{LiCl}$ 存在下, 通过 镁和多取代芳基溴代物的直接氧化插入反应可以在低 温 $\left(-10{ }^{\circ} \mathrm{C}\right)$ 条件下高效地合成目标格氏试剂, 进而用于 和氮杂环丙烷的开环反应(Scheme 40). 正是由于氯化
锂的存在, 格氏试剂的合成与反应得以在低温下进行, 为良好的官能团兼容性创造了条件.
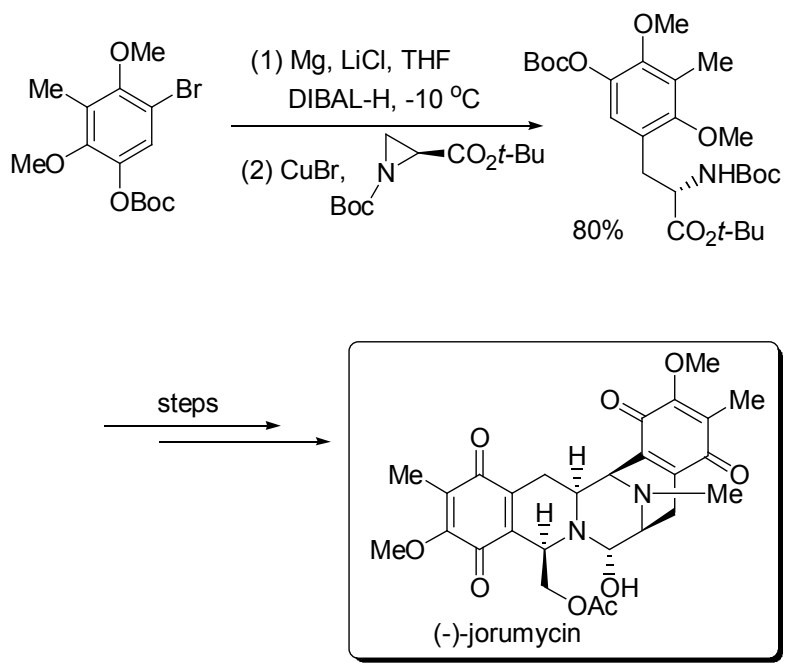

Scheme 40

最近, Fukuyama 等 ${ }^{[81]}$ 报道了以脯氨酸为起始原料, 以不对称分子内 Heck 反应为主要合成策略, 完成了 spirotry-rostatin A 的不对称全合成. 在合成 Heck 反应的 前体时，含氯化锂的芳基格氏试剂可以和醛在低温条件 下进行加成反应，体现出很好的反应活性、选择性和官 能团兼容性(Scheme 41). Fukuyama、Castle 和祝介平小 组的全合成工作表明：即使在复杂天然产物的全合成 中, 含氯化锂的格氏试剂同样具有很好的研究和应用前 景.

\section{4 结语与展望}

在过去数年的时间里, Knochel 小组以氯化锂为添 加剂, 较好地解决了困扰有机合成化学家关于含多能团 格氏试剂的合成问题. 然而通过上面综述也可以发现, $\mathrm{LiCl}$ 参与的格氏试剂的合成化学仍有较多问题函待解 决, 也有望成为有机镁化学研究的新方向. (1)经济性和 实用性. 相比于其它锂盐, $\mathrm{LiCl}$ 具有较好的原子经济性, 


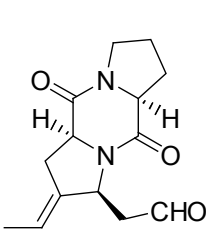

(1)

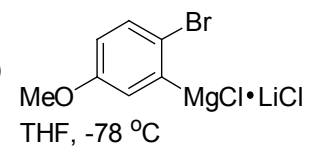

(2) Dess-Martin periodinane, $\mathrm{CH}_{2} \mathrm{Cl}_{2}, 84 \%$

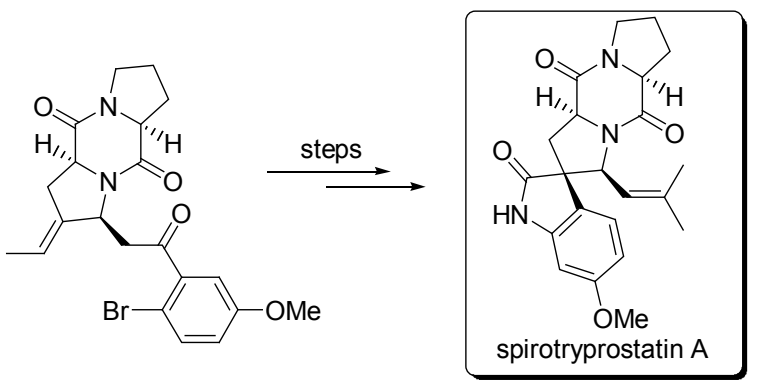

Scheme 41

但无水氯化锂价格并不便宜, 而且氯化锂往往需要超过 卤代烃的当量数; 与此同时, 即使在 $\mathrm{LiCl}$ 存在下, 经济 性较好的氯代烃应用还比较少, 这些都不利于该类反应 大规模开发应用. 此外, 该盐极易吸潮, 操作上存在较 大不便, 而且反应条件比较苛刻, 实际应用中存在较多 限制. (2)简易且新颖的 Knochel-Hauser 碱合成方法. 已 报道的 Knochel-Hauser碱合成往往通过四甲基哌啶和异 丙基氯化镁氯化锂置换反应加以制备, 合成过程中需要 两次标定有机镁试剂, 比较繁琐 ${ }^{[82]}$. 而相应的锌试剂则 可通过氯代四甲基哌啶与锌粉直接合成, 相对要简单得 多 ${ }^{[83]}$. 因此, 发展简易且高效的合成含氯化锂胺基镁试 剂的方法, 有利于进一步推广 Knochel-Hauser 碱在合成 中的应用. (3)稳定型格氏试剂的合成. 由于格氏试剂的 稳定性较差, 所以在实际使用时最好现时制备, 对反应 设备和条件要求较高. 最近, Knochel 等小组 ${ }^{[84]}$ 在稳定 型有机锌试剂方面的研究取得了突破, 为相应稳定型格 氏试剂的制备提供了参考. 稳定型格氏试剂的合成与发 展将会简化格氏试剂合成与应用的实验条件, 有望为格 氏试剂的合成与应用再次带来新的研究热潮. (4)手性格 氏试剂的合成 ${ }^{[85]}$. 与手性有机锂试剂的合成研究相 比 $^{[86]}$, 手性有机镁试剂的研究相对滞后. 为此, 开展手 性格氏试剂的合成将会是日后有机镁化学研究的重点 和热点. (5)格氏反应机理的研究 ${ }^{[12,87]}$. 目前, 关于氯化 锂在格氏反应中的促进机制研究还很少 ${ }^{[10,88]}$, 从而限制 了更好添加剂的篮选. 因此, 开展以 $\mathrm{LiCl}$ 作用机制的相 关研究将是进一步拓展格氏试剂合成及应用的关键课 题. (6)含氯化锂有机镁试剂在新反应中的应用. 例如, Tokuyama 小组 ${ }^{[89]}$ 利用 Knochel-Hauser 碱, 以溴化氢消 除反应得到的苯炔类化合物为中间体, 合成了一系列吲 哚啉和咔唑衍生物, 并成功应用于天然产物 Hepta- phylline 的合成. (7)新化学合成技术在格氏反应中的应

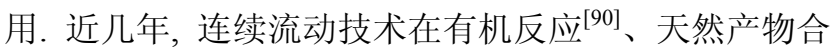
成 ${ }^{[91]}$ 、制药化学 ${ }^{[92]}$ 等领域得到了广泛的应用, 其中就包 括 Ley 和 Knochel 联合开展的连续流动技术在格氏反应 中的应用 ${ }^{[93]}$. 以上这些相关格氏试剂研究领域的持续 开展, 必将推动有机镁化学在有机合成、高分子材料化 学、化学反应工程中的进一步应用!

\section{References}

[1] (a) Grignard, V. C. R. Hebd. Séances Acad. Sci. 1900, 130, 1322. (b) Grignard, V. Ph.D. Dissertation, University of Lyon, Lyon, France, 1901.

[2] Knochel, P.; Krasovskiy, A.; Sapountzis, I. In Handbook of Functionalized Organometallics, Vol. 1, Ed.: Knochel, P., Wiley-VCH, Weinheim, 2005, 109.

(b) Mulvey, R. E. Organometallics 2006, 25, 1060.

[3] (a) Tamao, K.; Sumitani, K.; Kumada, M. J. Am. Chem. Soc. 1972, 94, 4374.

(b) Corriu, R. J. P.; Masse, J. P. J. Chem. Soc., Chem. Commun. 1972, 144.

(c) Seyferth, D. Organometallics 2009, 28, 1598.

[4] Boudier, A.; Bromm, L. O.; Lotz, M.; Knochel, P. Angew. Chem., Int. Ed. 2000, 39, 4415.

[5] (a) Knochel, P.; Dohle, W.; Gommermann, N.; Kneisel, F. F.; Kopp, F.; Korn, T.; Sapountzis, I.; Vu, V. A. Angew. Chem., Int. Ed. 2003, 42, 4302.

(b) Ila, H.; Baron, O.; Wagner, A. J.; Knochel, P. Chem. Commun. 2006, 583 .

(c) Ila, H.; Baron, O.; Wagner, A. J.; Knochel, P. Chem. Lett. 2006, 35,2 .

(d) Fang, H.; Xi, Z. Chemsitry 2005, 68, 8 (in Chinese).

(方红云, 席振峰, 化学通报, 2005, 68, 8.)

(e) Inoue, A.; Kitagawa, K.; Shinokubo, H.; Oshima, K. J. Org. Chem. 2001, 66, 4333.

(f) Bayh, O.; Awad, H.; Mongin, F.; Hoarau, C.; Trécourt, F.; Quéguiner, G.; Marsais, F.; Blanco, F.; Abarca, B.; Ballesteros, R. Tetrahedron 2005, 61, 4779.

(g) Reichle, M. A.; Breit, B. Angew. Chem., Int. Ed. 2012, 51, 5730 .

[6] (a) Knochel, P.; Schade M. A.; Bernhardt, S.; Manolikakes, G.; Metzger, A.; Piller, F. M.; Rohbogner, C. J.; Mosrin, M. Beilstein J. Org. Chem. 2011, 7, 1261.

(b) Haag, B. A.; Mosrin, M.; Ila, H.; Malakhov, V.; Knochel, P. Angew. Chem., Int. Ed. 2011, 50, 9794.

(c) Zhao, J.; Wan, H.; Gu, Y.; Du, Y.; Lv, J. Chem. Reag. 2013, 35, 27 (in Chinese).

(赵娟, 万洪, 谷玉杰, 杜咏梅, 吕剑, 化学试剂, 2013, 35, 27.)

(d) Krasovskiy, A.; Malakhov, V.; Gavryushin, A.; Knochel, P. Angew. Chem., Int. Ed. 2006, 45, 6040.

(e) Blümke, T.; Chen, Y.-H.; Peng, Z.; Knochel, P. Nat. Chem. 2010, 2, 313 .

(f) Metzger, A.; Schade, M. A.; Knochel, P. Org. Lett. 2008, 10, 1107.

(g) Peng, Z.; Konchel, P. Org. Lett. 2011, 13, 3198.

[7] Prévost, C. Bull. Soc. Chim. Fr. 1931, 49, 1372.

[8] Tamborski, C.; Moore, G. J. J. Organomet. Chem. 1971, 26, 153.

[9] Boymond, L.; Rottländer, M.; Cahiez, G.; Knochel, P. Angew. Chem., Int. Ed. 1998, 37, 1701.

[10] Kneisel, F. F.; Dochnahl, M.; Knochel, P. Angew. Chem., Int. Ed. 
2004, 43, 1017.

[11] Krasovskiy, A.; Knochel, P. Angew. Chem., Int. Ed. 2004, 43, 3333.

[12] Krasovskiy, A.; Straub, B. F.; Knochel, P. Angew. Chem., Int. Ed. 2006, 45, 159.

[13] Ren, H.; Krasovskiy, A.; Knochel, P. Org. Lett. 2004, 6, 4215.

[14] (a) Rauhut, C. B.; Vu, V. A.; Fleming, F. F.; Knochel, P. Org. Lett. 2008, 10, 1187.

(b) Nishimura, R. H. V.; Toledo, F. T.; Lopes, J. L. C.; Clososki, G. C. Tetrahedron Lett. 2013, 54, 287.

[15] (a) Ogawa, S.; Furukawa, N. J. Org. Chem. 1991, 56, 5723.

(b) Capozzi, M. A. M.; Cardellicchio, C.; Naso, F.; Spina, G.; Tortorella, P. J. Org. Chem. 2001, 66, 5933.

(c) Yamamoto, T.; Ogawa, S.; Sugawara, M.; Kawai, Y.; Sato, R. Bull. Chem. Soc. Jpn. 2006, 79, 460.

[16] (a) Rauhut, C. B.; Melzig, L.; Knochel, P. Org. Lett. 2008, 10, 3891 .

(b) Melzig, L.; Rauhut, C. B.; Knochel, P. Chem. Commun. 2009, 3536 .

[17] (a) Boudet, N.; Knochel, P. Org. Lett. 2006, 8, 3737.

(b) Boudet, N.; Lachs, J. R.; Knochel, P. Org. Lett. 2007, 9, 5525.

(c) Dubbaka, S. R.; Kienle, M.; Mayr, H.; Knochel, P. Angew. Chem., Int. Ed. 2007, 46, 9093.

(d) Duan, X.-F.; Ma, Z.-Q.; Zhang, F.; Zhang, Z.-B. J. Org. Chem. 2009, 74, 939 .

[18] Unsinn, A.; Dunst, C.; Knochel, P. Beilstein J. Org. Chem. 2012, 8, 2202.

[19] Hauser, C. R.; Walker, H. G. J. Am. Chem. Soc. 1947, 69, 295.

[20] Eaton, P. E.; Lee, C.-H.; Xiong, Y. J. Am. Chem. Soc. 1989, 111, 8016.

[21] (a) Eaton, P. E.; Lukin, K. A. J. Am. Chem. Soc. 1993, 115, 11370. (b) Zhang, M.-X.; Eaton, P. E. Angew. Chem., Int. Ed. 2002, 41, 2169.

[22] Krasovskiy, A.; Krasovskaya, V.; Knochel, P. Angew. Chem., Int. Ed. 2006, 45, 2958.

[23] Gu, Y. G.; Bayburt, E. K. Tetrahedron Lett. 1996, 37, 2565.

[24] Clososki, G. C.; Rohbogner, C. J.; Knochel, P. Angew. Chem., Int. Ed. 2007, 46, 7681 .

[25] Unsinn, A.; Rohbogner C. J.; Knochel, P. Adv. Synth. Catal. 2013, $355,1553$.

[26] (a) Wencel-Delord, J.; Dröge, T.; Liu, F.; Glorius, F. Chem. Soc. Rev. 2011, 40, 4740 .

(b) Arockiam, P. B.; Bruneau, C.; Dixneuf, P. H. Chem. Rev. 2012, 112, 5879.

(c) Yamaguchi, J.; Yamaguchi, A. D.; Itami, K. Angew. Chem., Int. Ed. 2012, 51, 8960.

[27] (a) Snieckus, V. Chem. Rev. 1990, 90, 879.

(b) Boudet, N.; Sase, S.; Sinha, P.; Liu, C.-Y.; Krasovskiy, A.; Knochel, P. J. Am. Chem. Soc. 2007, 129, 12358.

[28] (a) Rohbogner, C. J.; Clososki, G. C.; Knochel, P. Angew. Chem., Int. Ed. 2008, 47, 1503.

(b) Bellamy, E.; Bayh, O.; Hoarau, C.; Trécourt, F.; Quéguiner, G.; Marsais, F. Chem. Commun. 2010, 46, 7043.

(c) Rohbogner, C. J.; Wirth, S.; Knochel, P. Org. Lett. 2010, 12, 1984.

[29] (a) Barl, N. M.; Sansiaume-Dagousset, E.; Karaghiosoff, K.; Knochel, P. Angew. Chem., Int. Ed. 2013, 52, 10093.

(b) Sämann, C.; Coya, E.; Knochel, P. Angew. Chem., Int. Ed. 2014, 53, 1430 .

[30] Jaric, M.; Haag, B. A.; Unsinn, A.; Karaghiosoff, K.; Knochel, P. Angew. Chem., Int. Ed. 2010, 49, 5451.

[31] (a) Mosrin, M.; Knochel, P. Chem. Eur. J. 2009, 15, 1468.

(b) Jaric, M.; Haag, B. A.; Manolikakes, S. M.; Knochel, P. Org.
Lett. 2011, 13, 2306

(c) Groll, K.; Manolikakes, S. M.; du Jourdin, X. M.; Jaric, M.; Bredihhin, A.; Karaghiosoff, K.; Carell, T.; Knochel, P. Angew. Chem., Int. Ed. 2013, 52, 6776.

(d) Klatt, T.; Roman, D. S.; León, T.; Knochel, P. Org. Lett. 2014, $15,1232$.

[32] (a) Piller, F. M.; Bresser, T.; Fischer, M. K.; Knochel, P. J. Org. Chem. 2010, 75, 4365 .

(b) Bresser, T.; Knochel, P. Angew. Chem., Int. Ed. 2011, 50, 1914.

[33] Duez, S.; Steib, A. K.; Knochel, P. Org. Lett. 2012, 14, 1951.

[34] Piller, F. M.; Appukkuttan, P.; Gavryushin, A.; Helm, M.; Knochel, P. Angew. Chem., Int. Ed. 2008, 47, 6802.

[35] Blümke, T. D.; Piller, F. M.; Knochel, P. Chem. Commun. 2010, 46, 4082.

[36] (a) Kopp, F.; Knochel, P. Org. Lett. 2007, 9, 1639.

(b) Piller, F. M.; Knochel, P. Org. Lett. 2009, 11, 445.

(c) Despotopoulou, C.; Klier, L.; Knochel, P. Org. Lett. 2009, 11, 3326.

(d) Mosrin, M.; Bresser, T.; Knochel, P. Org. Lett. 2009, 11, 3406.

(e) Hass, D.; Mosrin, M.; Knochel, P. Org. Lett. 2013, 15, 6162.

[37] (a) Mosrin, M.; Knochel, P. Org. Lett. 2008, 10, 2497.

(b) Hawkins, V. F.; Wilkinson, M. C.; Whiting, M. Org. Process Res. Dev. 2008, 12, 1265.

(c) Tang, W.; Sarvestani, M.; Wei, X.; Nummy, L. J.; Patel, N.; Narayanan, B.; Byrne, D.; Lee, H.; Yee, N. K.; Senanayake, C. H. Org. Process Res. Dev. 2009, 13, 1426.

(d) Leermann, T.; Leroux, F. R.; Colobert, F. Org. Lett. 2011, 13, 4479.

(e) Demory, E.; Blandin, V.; Einhorn, J.; Chavant, P. Y. Org. Process Res. Dev. 2011, 15, 710.

(f) Anbarasan, P.; Neumann, H.; Beller, M. Chem. Eur. J. 2011, 17, 4217.

(g) Dunst, C.; Knochel, P. J. Org. Chem. 2011, 76, 6972.

[38] (a) Yamada, S.; Gavryushin, A.; Knochel, P. Angew. Chem., Int. Ed. 2010, 49, 2215.

(b) Yamada, S.; Knochel, P. Synthesis 2010, 2490.

[39] Anbarasan, P.; Neumann, H.; Beller, M. Angew. Chem., Int. Ed. 2010, 49, 2219.

[40] (a) Wong, K.-T.; Chien, Y.-Y.; Liao, Y.-L.; Lin, C.-C.; Chou, M.-Y.; Leung, M. J. Org. Chem. 2002, 67, 1041.

(b) Pintaric, C.; Olivero, S.; Gimbert, Y.; Chavant, P. Y.; Duñach, E. J. Am. Chem. Soc. 2010, 132, 11825.

[41] Haag, B. A.; Sämann, C.; Jana, A.; Knochel, P. Angew. Chem., Int. Ed. 2011, 50, 7290.

[42] (a) Miyaura, N.; Suzuki, A. Chem. Rev. 1995, 95, 2457.

(b) Bellina, F.; Carpita, A.; Rossi, R. Synthesis 2004, 2419.

(c) Martin R.; Buchwald, S. L. Acc. Chem. Res. 2008, 41, 1461.

[43] Heravi, M. M.; Hashemi, E.; Azimian, F. Tetrahedron 2014, 70, 7.

[44] Negishi, E. Angew. Chem., Int. Ed. 2011, 50, 6738.

[45] (a) Yin, L.; Liebscher, J. Chem. Rev. 2007, 107, 133.

(b) Slagt, V. F.; de Vries, A. H. M.; de Vries, J. G.; Kellogg, R. M. Org. Process Res. Dev. 2010, 14, 30.

(c) Johansson Seechurn, C. C. C.; Kitching, M. O.; Colacot, T. J.; Sineckus, V. Angew. Chem., Int. Ed. 2012, 51, 5062.

[46] Martin, R.; Buchwald, S. L. J. Am. Chem. Soc. 2007, 129, 3844.

[47] (a) Bolm, C.; Legros, J.; Le Piah, J.; Zani, L. Chem. Rev. 2004, 104, 217.

(b) Sherry, B. D.; Fürstner, A. Acc. Chem. Res. 2008, 41, 1500.

(c) Cahiez, A.; Moyeux, A. Chem. Rev. 2010, 110, 1435.

(d) Kuzmina, O. M.; Steib, A. K.; Flubacher, D.; Knochel, P. Org. Lett. 2012, 14, 4818.

(e) Steib, A. K.; Kuzmina, O. M.; Fernandez, S.; Flubacher, D.; 
Knochel, P. J. Am. Chem. Soc. 2013, 135, 15346.

[48] Kuzmina, O. M.; Steib, A. K.; Markiewicz, J. T.; Flubacher, D.; Knochel, P. Angew. Chem., Int. Ed. 2013, 52, 4945.

[49] (a) Hegedus, L. S.; Thompson, H. P. J. Am. Chem. Soc. 1985, 107, 5663.

(b) Terao, J.; Watanabe, H.; Ikumi, A.; Kuniyasu, H.; Kambe, N. $J$. Am. Chem. Soc. 2002, 124, 4222.

[50] Manolikakes G.; Knochel, P. Angew. Chem., Int. Ed. 2009, 48, 205.

[51] Kienle, M.; Knochel, P. Org. Lett. 2010, 12, 2702.

[52] Kofink, C. C.; Knochel, P. Org. Lett. 2006, 8, 4121.

[53] (a) Maji, M. S.; Pfeifer, T.; Studer, A. Angew. Chem., Int. Ed. 2008, 47, 9547.

(b) Kiefer, G.; Jeanbourquin, L.; Severin, K. Angew. Chem., Int. Ed. 2013, 52, 6302.

[54] Krasovskiy, A.; Tishkov, A.; del Amo, V.; Mayr, H.; Knochel, P. Angew. Chem., Int. Ed. 2006, 45, 5010.

[55] (a) Schade, M. A.; Manolikakes, G.; Knochel, P. Org. Lett. 2010, 12,3648 .

(b) Kienle, M.; Knochel, P. Org. Lett. 2010, 12, 2702.

(c) Sämann, C.; Schade, M. A.; Yamada, S.; Knochel, P. Angew. Chem., Int. Ed. 2013, 52, 9495.

[56] (a) Boudet, N.; Dubbaka, S. R.; Knochel, P. Org. Lett. 2008, 10, 1715 .

(b) Frischmuth, A.; Knochel, P. Angew. Chem., Int. Ed. 2013, 52, 10084.

[57] Bernhardt, S.; Shen, Z.-L.; Knochel, P. Chem. Eur. J. 2013, 19 , 828.

[58] Dong, Z.; Clososki, G. C.; Wunderlich, S. H.; Unsinn, A.; Li, J.; Knochel, P. Chem. Eur. J. 2009, 15, 457.

[59] a) Wunderlich, S. H.; Knochel, P. Angew. Chem., Int. Ed. 2007, 46, 7685 .

(b) Wunderlich, S. H.; Knochel, P. Chem. Commun. 2008, 47, 6387.

(c) Wunderlich, S. H.; Knochel, P. Org. Lett. 2008, 10, 4705.

(d) Mosrin, P.; Knochel, P. Org. Lett. 2009, 11, 1837.

(e) Wunderlich, S. H.; Rohbogner, C. J.; Unsinn, A.; Knochel, P. Org. Process Res. Dev. 2010, 14, 339.

[60] Wunderlich, S. H.; Knochel, P. Angew. Chem., Int. Ed. 2009, 48, 9717.

[61] Wunderlich, S. H.; Kienle, M.; Knochel, P. Angew. Chem., Int. Ed. 2009, 48, 7256.

[62] Jeganmohan, M.; Knochel, P. Angew. Chem., Int. Ed. 2010, 49, 8520.

[63] Wunderlich, S. H.; Knochel, P. Chem. Eur. J. 2010, 16, 3304.

[64] Ortiz, R. P.; Facchetti, A.; Marks, T. J. Chem. Rev. 2010, 110, 205.

[65] Yazaki, S.; Funahashi, M.; Kagimoto, J.; Ohno, H.; Kato, T. J. Am. Chem. Soc. 2010, 132, 7702

[66] Mishra, A.; Ma, C.-Q.; Bäuerle, P. Chem. Rev. 2009, 109, 1141.

[67] (a) Osaka, I.; McCullough, R. D. Acc. Chem. Res. 2008, 41, 1202. (b) Yokozawa, T.; Yokoyama, A. Chem. Rev. 2009, 109, 5595.

[68] (a) Tanaka, S.; Tamba, S.; Tanaka, D.; Sugie, A.; Mori, A. J. Am. Chem. Soc. 2011, 133, 16734.

(b) Tamba, S.; Mitsuda, S.; Tanaka, F.; Sugie, A.; Mori, A. Organometallics 2012, 31, 2263.

[69] (a) McCullough, R. D. Adv. Mater. 1998, 10, 93. (b) Huang, L.; Wu, S.; Qu, Y.; Geng, Y.; Wang, F. Macromolecules 2008, 41, 8944.

[70] Loewe, R. S.; Khersonsky, S. M.; McCullough, R. D. Adv. Mater. $1999,11,250$

[71] Miyakoshi, R.; Shimono, K.; Yokoyama, A.; Yokozawa, T. J. Am. Chem. Soc. 2006, 128, 16012.

[72] Stefan, M. C.; Javier, A. E.; Osaka, I.; McCullough, R. D. Macro- molecules 2009, 42, 30 .

[73] Maji, M. S.; Pfeifer, T.; Studer, A. Chem. Eur. J. 2010, 16, 5872.

[74] Chen, Q.; du Jourdin, X. M.; Knochel, P. J. Am. Chem. Soc. 2013, 135,4958

[75] (a) Nakao, Y.; Yamada, Y.; Kashihara, N.; Hiyama, T. J. Am. Chem. Soc. 2010, 132, 13666.

(b) Wasa, M.; Worrell, B. T.; Yu, J.-Q. Angew. Chem., Int. Ed. 2010, 49, 1275.

(c) Goriya, Y.; Ramana, C. V. Chem. Eur. J. 2012, 18, 13288.

[76] Haag, B. A.; Zhang, Z.-G.; Li, J.-S.; Knochel, P. Angew. Chem., Int. Ed. 2010, 49, 9513.

[77] Frischmuth, A.; Unsinn, A.; Groll, K.; Stadtmüller, H.; Knochel, P. Chem. Eur. J. 2012, 18, 10234

[78] (a) Hartwig, J. F. Angew. Chem., Int. Ed. 1998, 37, 2047.

(b) Surry, D. S.; Buchwald, S. L. Angew. Chem., Int. Ed. 2008, 47, 6338.

(c) Surry, D. S.; Buchwald, S. L. Chem. Sci. 2011, 2, 27.

(d) Sadig, J. E. R.; Willis, M. C. Synthesis 2011, 1.

[79] (a) Li, F.; Castle, S. L. Org. Lett. 2007, 9, 4033.

(b) Li, F.; Tartakoff, S. S.; Castle, S. L. J. Am. Chem. Soc. 2009, 131, 6674.

[80] Wu, Y.-C.; Zhu, J. Org. Lett. 2009, 11, 5558.

[81] Kitahara, K.; Shimokawa, J.; Fukuyama, T. Chem. Sci. 2013, 5, 904.

[82] Krasovskiy, A.; Knochel, P. Synthesis 2006, 890.

[83] Unsinn, A.; Ford, M. J.; Knochel, P. Org. Lett. 2013, 15, 1128.

[84] (a) Bernhardt, S.; Manolikakes, G.; Kunz, T.; Knochel, P. Angew. Chem., Int. Ed. 2011, 50, 9205.

(b) Stathakis, C. I.; Bernhardt, S.; Quint, V.; Knochel, P. Angew. Chem., Int. Ed. 2012, 51, 9428.

(c) Stathakis, C. I.; Manolikakes, S. M.; Knochel, P. Org. Lett. 2013, 15, 1302.

(d) Colombe, J. R.; Bernhardt, S.; Stathakis, C.; Buchwald, S. L.; Knochel, P. Org. Lett. 2013, 15, 5754.

[85] (a) Kaino, M.; Ishihara, K.; Yamamoto, H. Bull. Chem. Soc. Jpn. 1989, 62, 3736 .

(b) Hoffmann, R. W.; Hölzer, B.; Knopff, O.; Harms, K. Angew. Chem., Int. Ed. 2000, 39, 3072.

(c) Chen, Z.-N.; Fu, G.; Xu, X. Org. Lett. 2011, 13, 2046.

(d) Rayner, P. J.; O'Brien, P.; Horan, R. A. J. Am. Chem. Soc. 2013, 135, 8071

(e) Barker, G.; Alshawish, M. R.; Skilbeck, M. C.; Coldham, I. Angew. Chem., Int. Ed. 2013, 52, 7700.

[86] (a) Basu, A.; Thayumanavan, S. Angew. Chem., Int. Ed. 2002, 41, 716.

(b) Stymiest, J. L.; Bagutski, V.; French, R. M.; Aggarwal, V. K. Nature 2008, 456, 778.

(c) Lee, W. K.; Park, Y. S.; Beak, P. Acc. Chem. Res. 2009, 42, 224.

(d) Scott, H. K.; Aggarwal, V. K. Chem. Eur. J. 2011, 17, 13124.

(e) Dagousset, G.; Moriya, K.; Mose, R.; Berionni, G.; Karaghiosoff, K.; Knochel, P. Angew. Chem., Int. Ed. 2014, 53, 1425.

[87] (a) Hauk, D.; Lang, S.; Murso, A. Org. Process Res. Dev. 2006, 10, 733.

(b) Shi, L.; Chu, Y.; Knochel, P.; Mayr, H. J. Org. Chem. 2009, 74, 2760.

(c) Shi, L.; Chu, Y.; Knochel, P.; Mayr, H. Org. Lett. 2012, 14, 2602.

(d) García-Álvarez, P.; Graham, D. V.; Hevia, E.; Kennedy, A. R.; Klett, J.; Mulvey, R. E.; O'Hara, C. T.; Weatherstone, S. Angew. Chem., Int. Ed. 2008, 47, 8079.

[88] Kim, W.-J.; Ko, K.-Y.; Paik, S.-U.; Kim, H. Bull. Korean Chem. 
Soc. 1998, 9, 111.

[89] Noji, T.; Fujiwara, H.; Okano, K.; Tokuyama, H. Org. Lett. 2013, 15,1946 .

[90] (a) Sahoo, H. R.; Kralj, J. G.; Jensen, K. F. Angew. Chem., Int. Ed. 2007, 46, 5704.

(b) Webb, D.; Jamison, T. F. Chem. Sci. 2010, 1, 675.

(c) Wiles, C.; Watts, P. Green Chem. 2014, 16, 55.

[91] (a) Baxendale, I. R.; Deeley, J.; Griffiths-Jones, C. M.; Ley, S. V.; Saaby, S.; Tranmer, G. K. Chem. Commun. 2006, 2566.

(b) Baxendale, I. R.; Griffiths-Jones, C. M.; Ley, S. V.; Tranmer, G.
K. Synlett 2006, 427.

[92] Hopkin, M. D.; Baxendale, I. R.; Ley, S. V. Chem. Commun. 2010, $46,2450$.

[93] (a) Wakami, H.; Yoshida, J. Org. Process Res. Dev. 2005, 9, 787. (b) Nagaki, A.; Yamada, S.; Doi, M.; Tomida, Y.; Takabayashi, N.; Yoshida, J. Green Chem. 2011, 13, 1110.

(c) Brodmann, T.; Koos, P.; Metzger, A.; Knochel, P.; Ley, S. V. Green Chem. 2012, 14, 1335.

(d) Muñoz, J. M.; Alcázar, J.; de la Hoz, A.; Díaz-Ortiz, Á.; Alonso de Diegoa, S.-A. Green Chem. 2012, 14, 1335.

(Zhao, X.) 